\title{
Structured Synaptic Inhibition Has a Critical Role in Multiple-Choice Motion-Discrimination Tasks
}

\author{
Cheng Xue and $\mathbb{1}^{\circ}$ Feng Liu \\ National Laboratory of Solid State Microstructures and Department of Physics, Nanjing University, Nanjing 210093, China
}

\begin{abstract}
Neural network models have been constructed to explore the underlying neural mechanisms for decision-making in multiple-choice motion-discrimination tasks. Despite great progress made, several key experimental observations have not been interpreted. In contrast to homogeneous connectivity between pyramidal cells and interneurons in previous models, here their connectivity is totally structured in a continuous recurrent network model. Specifically, we assume two types of inhibitory connectivity: opposite-feature and similarfeature inhibition, representing that the connectivity strength has a maximum between neural pairs with opposite and identical preferred directions, respectively. With a common parameter set, the model accounted for a wide variety of physiological and behavioral data from monkey experiments, including those that previous models failed to reproduce. We found that the opposite-feature inhibition endows the decision-making circuit with an elimination strategy, which effectively reduces the number of choice alternatives for inspection to speed up the decision process at the cost of decision accuracy. Conversely, the similar-feature inhibition markedly enhances the ability of the network to make a choice among multiple options and improves the accuracy of decisions, while slowing down the decision process. A simplified mean-field model was also presented to analytically characterize the effect of structured inhibition on fine discrimination. We made a testable prediction: only the combination of cross-feature and similar-feature inhibition enables the circuit to make a categorical choice among 12 alternatives. Together, the current work highlights the importance of structured synaptic inhibition in multiple-choice decision-making processes and sheds light on the neural mechanisms for visual motion perception.
\end{abstract}

Key words: continuous recurrent network model; fine discrimination; motion discrimination; random-dot display; structured inhibition

\section{Introduction}

How cognitive functions are substantiated by anatomically stereotyped structures in the cerebral cortex is an essential issue in neuroscience. Continuous recurrent networks have been considered as promising substrates for various higher brain functions, including working memory (Compte et al., 2000), selective attention (Ardid et al., 2007, 2010), and perceptual decision-making (Furman and Wang, 2008). Typically, these networks are endowed with slow excitatory reverberation, which subserves the buildup and maintenance of persistent neural activity, and predominant feedback inhibition, which underlies the winner-takeall competition and attractor dynamics.

A series of modeling studies (Wang, 2002; Ma et al., 2006; Wong et al., 2007; Furman and Wang, 2008) was performed to explore the underlying mechanism for perceptual decisionmaking at the neuronal circuit level. Despite great progress made, some features of multiple-choice direction discrimination are

Received Jan. 1, 2014; revised July 14, 2014; accepted Aug. 16, 2014.

Author contributions: C.X. and F.L. designed research; C.X. and F.L. performed research; C.X. and F.L. contributed unpublished reagents/analytic tools; C.X. and F.L. analyzed data; C.X. and F.L. wrote the paper.

This work was supported by 973 Program Grant 2013CB834104, National Natural Science Foundation of China Grant 10604028, and the Priority Academic Program Development of Jiangsu Higher Education Institutions.

The authors declare no competing financial interests.

Correspondence should be addressed to Feng Liu, Department of Physics, Nanjing University, Nanjing 210093, China. E-mail: fliu@nju.edu.cn.

C. Xue's present address: Cognitive Neuroscience Laboratory, German Primate Center, Goettingen, Germany.

DOI:10.1523/JNEUROSCI.0001-14.2014

Copyright $\odot 2014$ the authors $\quad 0270-6474 / 14 / 3413444-14 \$ 15.00 / 0$ not yet interpreted. One feature may be associated with elimination process, a common tactic adopted to rule out the least likely options to reduce the number of alternatives for inspection. Although several psychological models were built to explain this process (Tversky, 1972; Yaniv and Schul, 1997; Roe et al., 2001), a biophysically realistic model based on neural circuitry is still lacking. Another feature relates to fine discrimination, i.e., the ability to make a categorical choice among similar options. Primates exhibit pronounced capabilities of fine discrimination (Weymouth, 1958; Purushothaman and Bradley, 2005); how this function is implemented physiologically remains elusive. For example, a recurrent network model with homogeneous inhibitory connectivity was built to simulate an eight-alternative motiondiscrimination task, but no categorical choices were made on $49 \%$ of simulation trials because of significant overlap in the neural representations of similar choice alternatives (Furman and Wang, 2008). Notably, the $45^{\circ}$ separation between adjacent targets is far greater than the discrimination threshold of primates (De Bruyn and Orban, 1988). It is important to probe whether and how fine discrimination can be accomplished in a local circuit.

It was shown that cortical interneurons also display feature selectivity similar to pyramidal neurons (Martin, 1988; Rao et al., 1999). This raises the possibility of reciprocal connections between pyramidal cells and interneurons based on their feature preference. Interestingly, various experimental studies demonstrated two forms of lateral inhibition: (1) one between neurons 
A
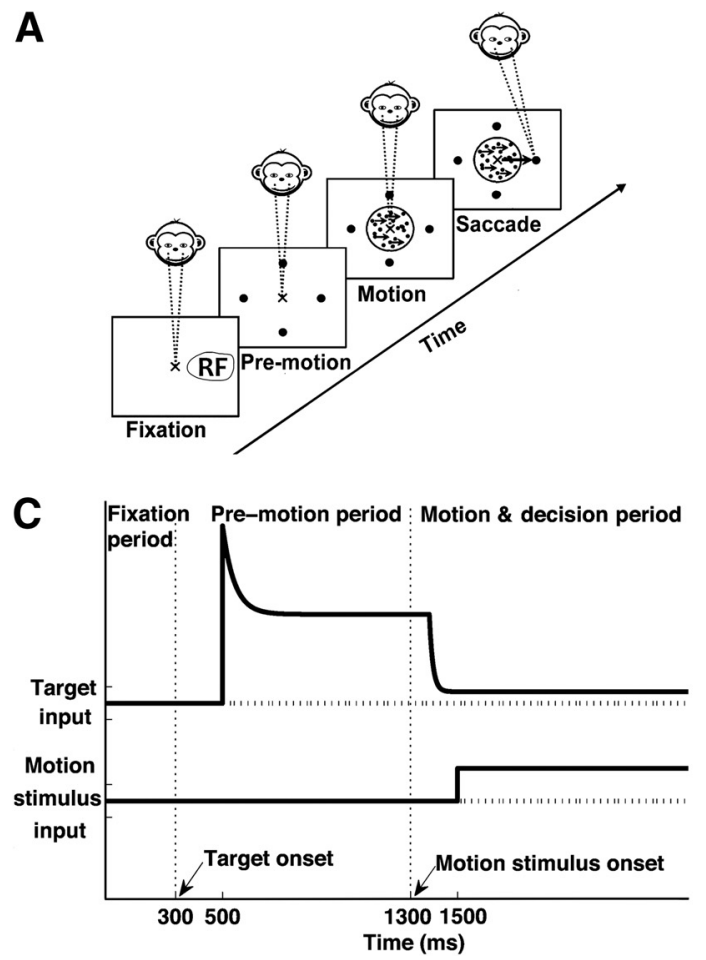

B

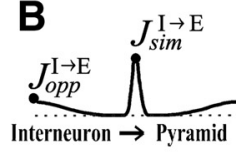

Interneuron $\rightarrow$ Interneuron

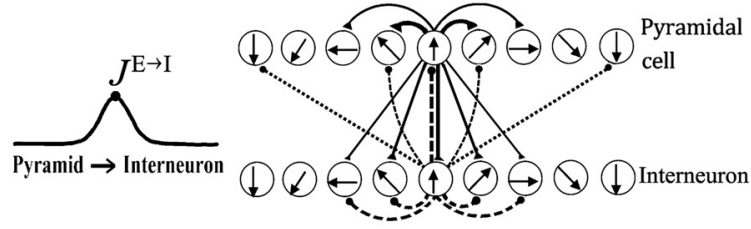

D

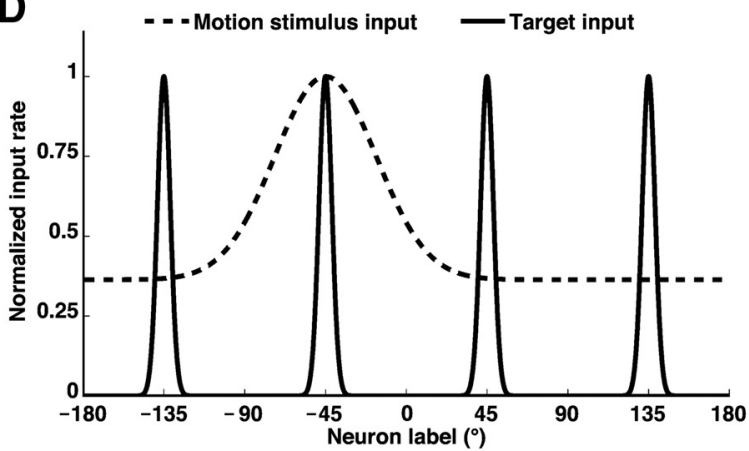

Figure 1. Network structure and simulation protocol. $A$, Schematics of a multiple-choice motion-discrimination task. A trial begins when a monkey fixates a central point on the monitor. Several targets then appear around the fixation point. After a delay, a noisy motion stimulus appears, and finally the monkey makes a saccade based on the coherent motion direction it perceives. $\boldsymbol{B}$, Synaptic connectivity within the network. The network consists of excitatory pyramidal cells and inhibitory interneurons. Each neuron is labeled by its preferred motion direction, which is uniformly distributed between $-180^{\circ}$ and $180^{\circ}$. The synaptic connectivity is heterogeneous except that between interneurons. The connectivity strength depends on the difference in preferred directions between neural pairs. Notably, there are two kinds of connectivity from interneurons to pyramidal cells, with a peak at $0^{\circ}$ difference (similar-feature inhibition) or $180^{\circ}$ difference (opposite-feature inhibition). C, D, Simulation protocol. Neurons also receive external synaptic currents representing sensory information about the targets and motion stimulus. The rates of presynaptic Poisson spike trains are characterized by the input signals. C, Schematics of time courses of the input signals. The targets are presented at $300 \mathrm{~ms}$, whereas the random-dot array is shown at $1300 \mathrm{~ms}$; both the inputs reach the network after a latency of $200 \mathrm{~ms}$. D, Normalized spatial profiles of the input signals as a function of preferred direction of each neuron.

with opposite feature preferences (Matsubara et al., 1987; Gilbert and Wiesel, 1989; Rao et al., 1999); and (2) the other between neurons with similar feature preferences (Martin, 1988; Ringach et al., 1997; Roerig and Kao, 1999; Neri and Levi, 2009). The former, called opposite-feature inhibition, may improve the contrast (or signal-to-noise ratio) of tuning curves of neurons, whereas the latter, called similar-feature inhibition, may sharpen tuning curves. It was also suggested that the two inhibitory mechanisms may contribute collectively to the implementation of higher cognitive functions (Martin, 1988; Rao et al., 1999). Indeed, we find that the elimination process and fine discrimination can be computationally reproduced once structured inhibition is included in a continuous circuit model. The model can account for experimental observations (Churchland et al., 2008), providing a computational framework for exploring multiple-choice motion discrimintaion.

\section{Materials and Methods}

To simulate the decision-making process in a multiple-alternative direction-discrimination task (Churchland et al., 2008), we extended a continuous recurrent circuit model (Furman and Wang, 2008) by introducing structured connectivity between excitatory and inhibitory neurons. A typical trial began when a monkey fixated a central point on the monitor (Fig. 1A). A number of choice targets then appeared in the periphery. One of the targets was within the response field of the recorded neuron. After a delay, a dynamic random-dot array appeared at the monitor center, where a fraction of dots moved coherently toward one of the targets while the others moved randomly in all other directions. The monkey reported the perceived motion direction by making a saccade to the corresponding target whenever it was ready. The model network consists of $N_{E}=2048$ pyramidal cells and $N_{I}=512$ interneurons. Neurons are spatially distributed according to the motion direction to which they are most sensitive; each cell is labeled by its preferred direction, which is uniformly distributed between $-180^{\circ}$ and $180^{\circ}$ (Fig. $\left.1 B\right)$.

Model setup. Both pyramidal cells and interneurons are modeled as leaky integrate-and-fire (LIF) neurons (Tuckwell, 1988). The subthreshold membrane potential $V_{m}(t)$ obeys

$$
C_{m} \frac{d V_{m}(t)}{d t}=-g_{L}\left(V_{m}-V_{L}\right)-I_{\text {syn }}(t)
$$

where $C_{m}$ is the membrane capacitance, $g_{L}$ is the leaky conductance, $V_{L}$ is the resting potential, and $I_{\text {syn }}$ is the total synaptic current. When $V_{m}$ reaches a threshold $V_{\text {th }}$, a spike is fired, and $V_{m}$ is reset to a potential $V_{\text {reset }}$ for a refractory period $\tau_{\text {ref }} C_{m}=0.5 \mathrm{nF}, g_{L}=25 \mathrm{nS}$, and $\tau_{\text {ref }}=2 \mathrm{~ms}$ for pyramidal cells; $C_{m}=0.2 \mathrm{nF}, g_{L}=20 \mathrm{nS}$, and $\tau_{\text {ref }}=1 \mathrm{~ms}$ for interneurons. $V_{L}=-70 \mathrm{mV}, V_{\text {reset }}=-55 \mathrm{mV}$, and $V_{\text {th }}=-50 \mathrm{mV}$ for all neurons.

Neurons receive both recurrent (from neurons within the network) and external synaptic inputs. The pyramid-to-pyramid, pyramid-tointerneuron, or interneuron-to-pyramid connectivity strength depends on their feature preference, whereas the interneuron-to-interneuron connectivity is homogeneous. EPSCs are mediated by AMPA receptors (AMPARs) and NMDA receptors (NMDARs), whereas IPSCs are mediated by $\mathrm{GABA}_{\mathrm{A}}$ receptors (GABARs). Neurons also receive external AMPAR-mediated currents representing sensory information about the visual targets and motion stimulus, as well as background synaptic input mimicking spontaneous activity outside the local network. Thus, the total synaptic current to pyramidal cell $i$ is given by 


$$
I_{i, \text { syn }}^{E}=\left(I_{i, \mathrm{AMPA}}^{E \rightarrow E}+I_{i, \mathrm{NMDA}}^{E \rightarrow E}+I_{i, \mathrm{GABA}}^{I \rightarrow E}\right)+I_{i, \mathrm{AMPA}}^{\mathrm{Ext} \rightarrow E}+I_{i, \mathrm{AMPA}}^{\mathrm{Back} \rightarrow E},
$$

where the first three terms denote recurrent inputs from excitatory $(E)$ and inhibitory $(I)$ neurons, $I_{i, \mathrm{AMPA}}^{\mathrm{Ext} \rightarrow E}$ represents the task-related input, and $I_{i, \mathrm{AMPA}}^{\mathrm{Back} \rightarrow E}$ denotes background noise. Similarly, the total synaptic current to interneuron $i$ is

$$
I_{i, \mathrm{syn}}^{I}=\left(I_{i, \mathrm{AMPA}}^{E \rightarrow I}+I_{i, \mathrm{NMDA}}^{E \rightarrow I}+I_{i, \mathrm{GABA}}^{I \rightarrow I}\right)+I_{i, \mathrm{AMPA}}^{\mathrm{Ext} \rightarrow I}+I_{i, \mathrm{AMPA}}^{\mathrm{Back} \rightarrow I} .
$$

The AMPAR-, NMDAR-, and GABAR-mediated currents are separately modeled by

$$
\begin{gathered}
I_{i, \mathrm{AMPA}}^{\alpha}(t)=g_{\mathrm{AMPA}}^{\alpha}\left(V_{i}(t)-V_{E}\right) \sum_{j=1}^{N_{E}} W_{i j}^{\alpha} S_{j}^{\mathrm{AMPA}}(t), \\
I_{i, \mathrm{MMDA}}^{\alpha}(t)=\frac{g_{\mathrm{NMDA}}^{\alpha}\left(V_{i}(t)-V_{E}\right)}{1+\left[\mathrm{Mg}^{2+}\right] \exp \left(-0.062 V_{i}(t) / 3.57\right)} \sum_{j=1}^{N_{E}} W_{i j}^{\alpha} S_{j}^{\mathrm{NMDA}}(t), \\
I_{i, \mathrm{GABA}}^{\beta}(t)=g_{\mathrm{GABA}}^{\beta}\left(V_{i}(t)-V_{I}\right) \sum_{j=1}^{N_{I}} W_{i j}^{\beta} S_{j}^{\mathrm{GABA}}(t),
\end{gathered}
$$

where $\alpha$ denotes $E \rightarrow E$ or $E \rightarrow I, \beta$ denotes $I \rightarrow E$ or $I \rightarrow I, g$ is the maximum synaptic conductance, $V_{E}=0 \mathrm{mV}$ and $V_{I}=-70 \mathrm{mV}$ are separately the reversal potentials of excitatory and inhibitory synapses, and $\left[\mathrm{Mg}^{2+}\right]=1 \mathrm{~mm}$ (Furman and Wang, 2008). The index $j$ runs over the presynaptic neurons, $W_{i j}$ is the connectivity strength, and $S_{j}$ is the gating variable (i.e., the fraction of receptors in the open state). The values of maximum conductance (in nS) are $g_{\mathrm{AMPA}}^{E \rightarrow E}=427.0 / N_{E}, g_{\mathrm{NMDA}}^{E} \rightarrow{ }^{E}=$ $1377.4 / N_{E}, g_{\mathrm{AMPA}}^{E \rightarrow I}=370.1 / N_{E}, g_{\mathrm{NMDA}}^{E} \rightarrow I=1197.5 / N_{E}, g_{\mathrm{GABA}}^{I \rightarrow E}=686.07 / N_{I}$, and $g_{\mathrm{GABA}}^{I \rightarrow I}=532.09 / N_{I}$. They are inversely proportional to the number of pyramidal cells or interneurons, which is to keep the total synaptic conductance unchanged when the network size is varied.

Given a spike train $\left\{t_{k}\right\}$ in the presynaptic neuron $j, S_{j}^{\mathrm{AMPA}}$ follows a fast dynamics:

$$
\frac{d S_{j}^{\mathrm{AMPA}}(t)}{d t}=-\frac{S_{j}^{\mathrm{AMPA}}}{\tau_{\mathrm{AMPA}}}+\sum_{k} \delta\left(t-t_{k}\right),
$$

with $\tau_{\mathrm{AMPA}}=2 \mathrm{~ms}$. Similarly, $S_{j}^{\mathrm{GABA}}$ is determined by

$$
\frac{d S_{j}^{\mathrm{GABA}}(t)}{d t}=-\frac{S_{j}^{\mathrm{GABA}}}{\tau_{\mathrm{GABA}}}+\sum_{k} \delta\left(t-t_{k}\right),
$$

with $\tau_{\mathrm{GABA}}=10 \mathrm{~ms}$. In contrast, $S_{j}^{\mathrm{NMDA}}$ obeys a slow dynamics:

$$
\begin{gathered}
\frac{d S_{j}^{\mathrm{NMDA}}(t)}{d t}=-\frac{S_{j}^{\mathrm{NMDA}}}{\tau_{\mathrm{NMDA}}}+\alpha_{\mathrm{NMDA}} x_{j}\left(1-S_{j}^{\mathrm{NMDA}}\right) \\
\text { and } \\
\frac{d x_{j}(t)}{d t}=-\frac{x_{j}}{\tau_{x}}+\sum_{k} \delta\left(t-t_{k}\right)
\end{gathered}
$$

where $x_{j}(t)$ is an intermediate gating variable, $\tau_{\mathrm{NMDA}}=100 \mathrm{~ms}, \alpha_{\mathrm{NMDA}}$ $=0.5 \mathrm{kHz}$, and $\tau_{x}=2 \mathrm{~ms}$.

For external input and background noise, each current item is given by $I_{i, \mathrm{AMPA}}=g\left(V_{i}-V_{E}\right) S_{i}$. $S_{i}$ obeys Equation 7, determined by a presynaptic Poisson spike train. For background noise, the values of maximum conductance are $g^{\text {Back } \rightarrow E}=2.9 \mathrm{nS}$ and $g^{\text {Back } \rightarrow I}=2.295 \mathrm{nS}$, and the rate of Poisson spike train is $v_{\text {back }}=1700 \mathrm{~Hz} /$ cell (for external inputs, see below, Simulation protocol).

With these parameter values, the network exhibits three prominent features. First, recurrent excitation is mainly mediated by NMDARs, so that slow reverberation underlies the temporal integration of sensory evidence. Here, NMDAR channels contribute $86 \%$ to recurrent excitatory charge entry at a holding potential of $-65 \mathrm{mV}$. Second, the network as a whole is dominated by recurrent inhibition, which enables the winner-take-all competition among neural representations of multiple alternatives. Third, neurons are subject to a large amount of background noise. These are important for a decision-making circuit (Furman and Wang, 2008).
Recurrent connectivity. The strength of synaptic connectivity between two pyramidal cells or between a pyramidal cell and an interneuron depends on the difference between their preferred directions, $\Delta \theta=\left|\theta_{i}-\theta_{j}\right|$. The dependence is Gaussian-like, i.e.,

$$
\begin{gathered}
W_{i j}^{E \rightarrow E}=W^{E \rightarrow E}(\Delta \theta)=J_{-}^{E \rightarrow E}+\left(J_{+}^{E \rightarrow E}-J_{-}^{E \rightarrow E}\right) \exp \left(-\frac{\Delta \theta^{2}}{2 \sigma_{E \rightarrow E}^{2}}\right), \\
W_{i j}^{E \rightarrow I}=W^{E \rightarrow I}(\Delta \theta)=J_{-}^{E \rightarrow I}+\left(J_{+}^{E \rightarrow I}-J_{-}^{E \rightarrow I}\right) \exp \left(-\frac{\Delta \theta^{2}}{2 \sigma_{E \rightarrow I}^{2}}\right),
\end{gathered}
$$

with $\sigma_{E \rightarrow E}=6.38^{\circ}$ and $\sigma_{E \rightarrow I}=42.8^{\circ}$, consistent with the assumption that the $E-I$ connectivity is broader than the $E-E$ connectivity (Edin et al., 2009), $J_{+}^{E} \rightarrow E=2.121$ and $J_{+}^{E} \rightarrow I=1.27$. The structured $E-E$ and $E-I$ connectivity is essential to reproduce the tuning curves of pyramidal cells and interneurons.

To implement both similar-feature and opposite-feature inhibition, we model the $I-E$ connectivity strength as follows:

$$
\begin{aligned}
W_{i j}^{I \rightarrow E}=W^{I \rightarrow E}(\Delta \theta)= & J_{-}^{I \rightarrow E}+\left(J_{+, \text {sim }}^{I \rightarrow E}-J_{-}^{I \rightarrow E}\right) \exp \left(-\frac{\Delta \theta^{2}}{2 \sigma_{I \rightarrow E, \text { sim }}^{2}}\right) \\
& +\left(J_{+, \text {opp }}^{I \rightarrow E}-J_{-}^{I \rightarrow E}\right) \exp \left(-\frac{\left(180^{\circ}-\Delta \theta\right)^{2}}{2 \sigma_{I \rightarrow E, \text { opp }}^{2}}\right),
\end{aligned}
$$

with $\sigma_{I \rightarrow E \text {,opp }}=60.0^{\circ}$ and $\sigma_{I \rightarrow E \text {,sim }}=5.0^{\circ}$, consistent with the argument that opposite-feature inhibition is much broader than similarfeature inhibition (Rao et al., 1999, 2000). $J_{+, \text {opp }}^{I \rightarrow E}$ and $J_{+, \text {sim }}^{I \rightarrow E}$ (abbreviated as $J_{\text {opp }}$ and $J_{\text {sim }}$ thereafter) are the only two free parameters in the model and may vary in different simulation protocols. In contrast, the I-I connectivity strength is uniform, i.e.,

$$
W_{i j}^{I \rightarrow I}=\frac{1}{N_{I}} .
$$

All the $J_{-}$values are determined by the normalization condition, i.e.,

$$
\frac{1}{360^{\circ}} \int_{0^{\circ}}^{360^{\circ}} W(\Delta \theta) d(\Delta \theta)=1 .
$$

The recurrent synapses have heterogeneous transmission latencies. The latencies of excitatory synapses are assumed to obey a Gaussian distribution with a mean \pm SD of $1.5 \pm 0.5 \mathrm{~ms}$ (Markram et al. 1997), whereas those of inhibitory synapses are drawn from a Gaussian distribution with a mean \pm SD of $0.3 \pm 0.1 \mathrm{~ms}$ (Bartos et al., 2001).

Simulation protocol. To simulate the multiple-alternative motiondiscrimination tasks (Churchland et al., 2008), we construct the following simulation protocol. The visual targets and the motion stimulus are represented by AMPAR-mediated synaptic currents. The external input to pyramidal cell $i$ is

$$
I_{i, \mathrm{AMPA}}^{\mathrm{Ext} \rightarrow E}(t)=I_{i, \mathrm{tar}}^{E}(t)+I_{i, \text { stim }}^{E}(t),
$$

with $I_{i, \text { tar }}^{E}=g_{\text {tar }}^{E}\left(V_{i}(t)-V_{E}\right) S_{i, \text { tar }}^{E}$ and $I_{i, \text { stim }}^{E}=g_{\text {stim }}^{E}\left(V_{i}(t)-V_{E}\right) S_{i, \text { stim }}^{E}$. $g_{\text {tar }}^{E}=14.5 \mu \mathrm{S}, g_{\text {stim }}^{E}=12.0 \mu \mathrm{S}$, and $S_{i, \text { tar }}^{E}$ and $S_{i, \text { stim }}^{E}$ obey Equation 7 , determined by independent Poisson spike trains with rates $v_{i, \text { tar }}^{E}$ and $v_{i, \text { stim }}^{E}$ as described below.

The target input projects selectively to pyramidal cells located around the directions of the targets. The rate $v_{i, \text { tar }}^{E}$ depends on the preferred direction $\theta_{i}$ of the pyramidal cell being targeted and varies with time (Furman and Wang, 2008):

$$
\nu_{i, \operatorname{tar}}^{E}=h(t) \sum_{k=1}^{n_{\text {tar }}} \exp \left(-\frac{\left(\theta_{i}-\theta_{k}^{\mathrm{tar}}\right)^{2}}{\sigma_{\mathrm{tar}}^{2}}\right),
$$

where $n_{\mathrm{tar}}$ is the number of targets, $\theta_{k}^{\mathrm{tar}}$ is the visual angle of the $k$ th target, and $\sigma_{\mathrm{tar}}=5^{\circ}$. Similar to that in previous studies (Wong et al., 2007; Furman and Wang, 2008), the time-dependent modulator $h(t)$ is described by 


$$
h(t)= \begin{cases}0 & 0<t<t_{1} \\ A_{1}+A_{2} e^{-\frac{t-t_{1}}{\tau_{1}}} & t_{1} \leq t \leq t_{2}+80 \\ A_{3}+\left(A_{1}-A_{3}\right) e^{-\frac{t-t_{2}-80}{\tau_{2}}} & t_{2}+80<t\end{cases}
$$

where $t_{0}=300 \mathrm{~ms}$ and $t_{2}=1300 \mathrm{~ms}$ represent the onset times for the targets and motion stimulus, respectively, and $t_{1}=t_{0}+200$ (since the signals are assumed to reach the network after a latency of $200 \mathrm{~ms}$; Fig. $1 C)$. The modulator $h(t)$ is to model the spike-rate adaptation of upstream neurons encoding the targets after the onset of targets and the presumed divided attention on stimulus onset. The parameters $A_{1}$ and $A_{2}$ control the magnitude of the target input before the motion onset, whereas $A_{3}$ determines the magnitude of the target input during the decision process and directly affects the psychometric functions. We use $\tau_{1}=50 \mathrm{~ms}, \tau_{2}=15 \mathrm{~ms}, A_{1}=272 \mathrm{~Hz}, A_{2}=381 \mathrm{~Hz}$, and $A_{3}=35 \mathrm{~Hz}$ to fit experimental data.

Based on the tuning curves of neurons in the middle temporal area (MT) in response to a random-dot display (Britten and Newsome, 1998), $v_{i, \text { stim }}^{E}$ is modeled as

$$
\nu_{i, \text { stim }}^{E}=r_{0}+c^{\prime}\left(-r_{1}+r_{2} \exp \left(-\frac{\left(\theta_{i}-\theta_{\text {stim }}\right)^{2}}{\sigma_{\text {stim }}^{2}}\right)\right),
$$

where $\theta_{\text {stim }}$ is the coherent motion direction and $c^{\prime}\left(0 \leq c^{\prime} \leq 1\right)$ denotes the motion coherence (Fig. 1D). We use $r_{0}=25 \mathrm{~Hz}, r_{1}=10 \mathrm{~Hz}, r_{2}=70$ $\mathrm{Hz}$, and $\sigma_{\text {stim }}=40^{\circ}$.

The interneurons receive one external input attributable to target presentation:

$$
I_{i, \mathrm{AMPA}}^{\mathrm{Ext} \rightarrow I}=I_{i, \operatorname{tar}}^{I}=g_{\mathrm{tar}}^{I}\left(V_{i}-V_{E}\right) S_{i, \mathrm{tar}}^{I} .
$$

The maximal conductance is $g_{\operatorname{tar}}^{I}=8.0 \mu \mathrm{S}$, and the presynaptic spike train is modeled as a Poisson process with rate $v_{i, \text { tar }}^{I}$, which varies with time but is independent of neuron label:

$$
\nu_{i, \text { tar }}^{I}(t)= \begin{cases}0 & 0<t<t_{1} \\ B_{1}+B_{2} e^{-\frac{t-t_{1}}{\tau_{1}}} & t_{1} \leq t \leq t_{2}+80 \\ B_{1} e^{-\frac{t-t_{2}-80}{\tau_{2}}} & t_{2}+80<t\end{cases}
$$

with $B_{1}=128 \mathrm{~Hz}$ and $B_{2}=179 \mathrm{~Hz}$. This is also to model the spike-rate adaptation in those upstream neurons after target appearance and divided attention after motion onset.

Simplified ring model. The biophysically realistic model allows a realtime simulation of synaptic dynamics, thus enabling a direct comparison of simulation results with electrophysiological data. However, it is far too complicated for analytical investigation of dynamic properties of the whole network. To analytically characterize the effect of structured inhibition on the attractor dynamics and decision behavior, we reduce the above spiking network model to a simplified ring model. The inhibition from interneurons is modeled as a negative term in the synaptic input, making it an instant feedback without time delay.

The activity of a pyramidal cell $i$ is represented solely by the gating variable of its NMDARs, $s_{i}$, whose dynamics are described by a differential equation (Wong and Wang, 2006; You and Wang, 2013):

$$
\frac{d s_{i}}{d t}=-\frac{s_{i}}{\tau_{s}}+\left(1-s_{i}\right) \alpha \phi\left(I_{i}\right),
$$

where $\alpha=0.5 \mathrm{kHz}, \tau_{\mathrm{s}}=100 \mathrm{~ms}$, and $\phi\left(I_{i}\right)$ represents the steady-state firing rate of the neuron when its input is a constant $I_{i}$. The relationship is given by (Abbott and Chance, 2005)

$$
\phi\left(I_{i}\right)=\frac{c I_{i}-I_{0}}{1-\exp \left(-g\left(c I_{i}-I_{0}\right)\right)}
$$

with $c=310(\mathrm{VnC})^{-1}, g=0.16 \mathrm{~s}$, and $I_{0}=125 \mathrm{~Hz}$. The synaptic input includes the recurrent and external inputs:

$$
I_{i}(t)=\sum_{j=1}^{N} W\left(\Delta_{i j}\right) s_{j}+I_{i}^{\mathrm{Ext}}
$$

with $\Delta_{i j}=\left\{\begin{array}{ll}|i-j| & |i-j|<\frac{N}{2} \\ \frac{N}{2}-|i-j| & |i-j| \geq \frac{N}{2}\end{array}\right.$. The input from neuron $j$ to neuron $i$ can be excitatory or inhibitory, depending on the sign of $W\left(\Delta_{i j}\right)$, which is defined as

$$
\begin{aligned}
& W\left(\Delta_{i j}\right)=g_{E}\left\{J_{-}^{E \rightarrow E}+\left(J_{+}^{E \rightarrow E}-J_{-}^{E \rightarrow E}\right) \exp \left(-\frac{\Delta_{i j}^{2}}{\sigma_{E}^{2}}\right)\right\}-g_{I}\left(J_{-}^{I \rightarrow E}+\left(J_{+, \text {sim }}^{I \rightarrow E}\right.\right. \\
& \left.\left.-J_{-}^{I \rightarrow E}\right) \exp \left(-\frac{\Delta_{i j}^{2}}{\sigma_{I, \text { sim }}^{2}}\right)+\left(J_{+, \text {opp }}^{I \rightarrow E}-J_{-}^{I \rightarrow E}\right) \exp \left(-\frac{\left(\frac{N}{2}-\Delta_{i j}\right)^{2}}{\sigma_{I, \text { opp }}^{2}}\right)\right\} . \quad(24)
\end{aligned}
$$

Thus, the excitatory and inhibitory inputs to cell $i$ are separately described by

$$
\begin{array}{r}
I_{i, E}(t)=\sum_{j=1}^{N} g_{E}\left\{J_{-}^{E \rightarrow E}+\left(J_{+}^{E \rightarrow E}-J_{-}^{E \rightarrow E}\right) \exp \left(-\frac{\Delta_{i j}^{2}}{\sigma_{E}^{2}}\right)\right\} s_{j} \\
I_{i, I}(t)=\sum_{j=1}^{N} g_{I}\left(J_{-}^{I \rightarrow E}+\left(J_{+, \mathrm{sim}}^{I \rightarrow E}-J_{-}^{I \rightarrow E}\right) \exp \left(-\frac{\Delta_{i j}^{2}}{\sigma_{I, \mathrm{sim}}^{2}}\right)+\left(J_{+, \mathrm{opp}}^{I \rightarrow \infty}\right.\right. \\
\left.\left(-J_{-}^{I \rightarrow E}\right) \exp \left(-\frac{\left(\frac{N}{2}-\Delta_{i j}\right)^{2}}{\sigma_{I, \mathrm{opp}}^{2}}\right)\right\}
\end{array}
$$

$g_{E}=7.0, g_{I}=7.3, N=512, \sigma_{E}=15, \sigma_{I, \text { sim }}=43, \sigma_{I, \text { opp }}=114$, and all the other parameters are the same as defined above. In plotting Figure 10B-E, the firing rate is calculated via assuming that a LIF neuron receives only an NMDAR-mediated synaptic current determined by $s_{i}$.

Numerical approximation of steady states. First, we use the NewtonRaphson method to determine the steady states. For any initial state vector $\mathbf{S}=\left\{s_{1}, s_{2}, \ldots, s_{N}\right\}$, we calculate its Jacobian matrix $J^{N \times N}$, whose elements are given by

$$
\begin{aligned}
J_{i j}=\frac{\partial}{\partial s_{j}} \frac{d s_{i}}{d t}= & -\left[\frac{1}{\tau_{s}}+\alpha \phi\left(\left.I_{i}\right|_{s}\right)\right] \delta_{i j}+\left.\left(1-s_{i}\right) \alpha \frac{d \phi\left(I_{i}\right)}{d I_{i}}\right|_{s} \frac{\partial I_{i}}{\partial s_{j}}= \\
& -\left[\frac{1}{\tau_{s}}+\alpha \phi\left(\left.I_{i}\right|_{s}\right)\right] \delta_{i j}+\left.\left(1-s_{i}\right) \alpha \frac{d \phi\left(I_{i}\right)}{d I_{i}}\right|_{s} W\left(\Delta_{i j}\right)
\end{aligned}
$$

with $\delta_{i j}=\left\{\begin{array}{ll}1 & i=j \\ 0 & i \neq j\end{array}\right.$. Then, the state vector $S$ is updated by

$$
S_{\text {next }}=S-\frac{d S}{d t} \times\left(J^{-1}\right)^{T},
$$

such that $S_{\text {next }}$ is closer to the steady state. The Jacobian matrix of $S_{\text {next }}$ is calculated to update $S_{\text {next }}$ in the same way. This loop goes on until the norm of $\mathrm{d} S / \mathrm{d} t$ is sufficiently small, and the fixed state vector is considered to represent a steady state $\left(S_{\mathrm{ss}}=S\right)$.

To examine the stability of the steady-state $S_{\mathrm{ss}}$, we solve the secular equations of the current Jacobian matrix $J_{\mathrm{ss}}$ :

$$
J_{\mathrm{SS}}^{N \times N} \delta S^{N \times 1}=\lambda \delta S^{N \times 1},
$$

where $\delta S$ denotes any eigenvalue perturbation of $S_{\text {eq }}$. If all the eigenvalues $\left\{\lambda_{1}, \lambda_{2}, \ldots, \lambda_{N}\right\}$ are negative, the steady state is stable; otherwise, it is unstable.

Numerical method. The integration method for ordinary differential equations is a modified second-order Runge-Kutta algorithm (Liu and Wang, 2008), with a time step of $0.1 \mathrm{~ms}$. Of note, we adopted the basic structure of the model of Furman and Wang (2008) but did not take the same value for each parameter. Given structured connectivity between pyramidal cells and interneurons, we had to adjust parameter values so as to reproduce the experimental data. We also analyzed the robustness of 

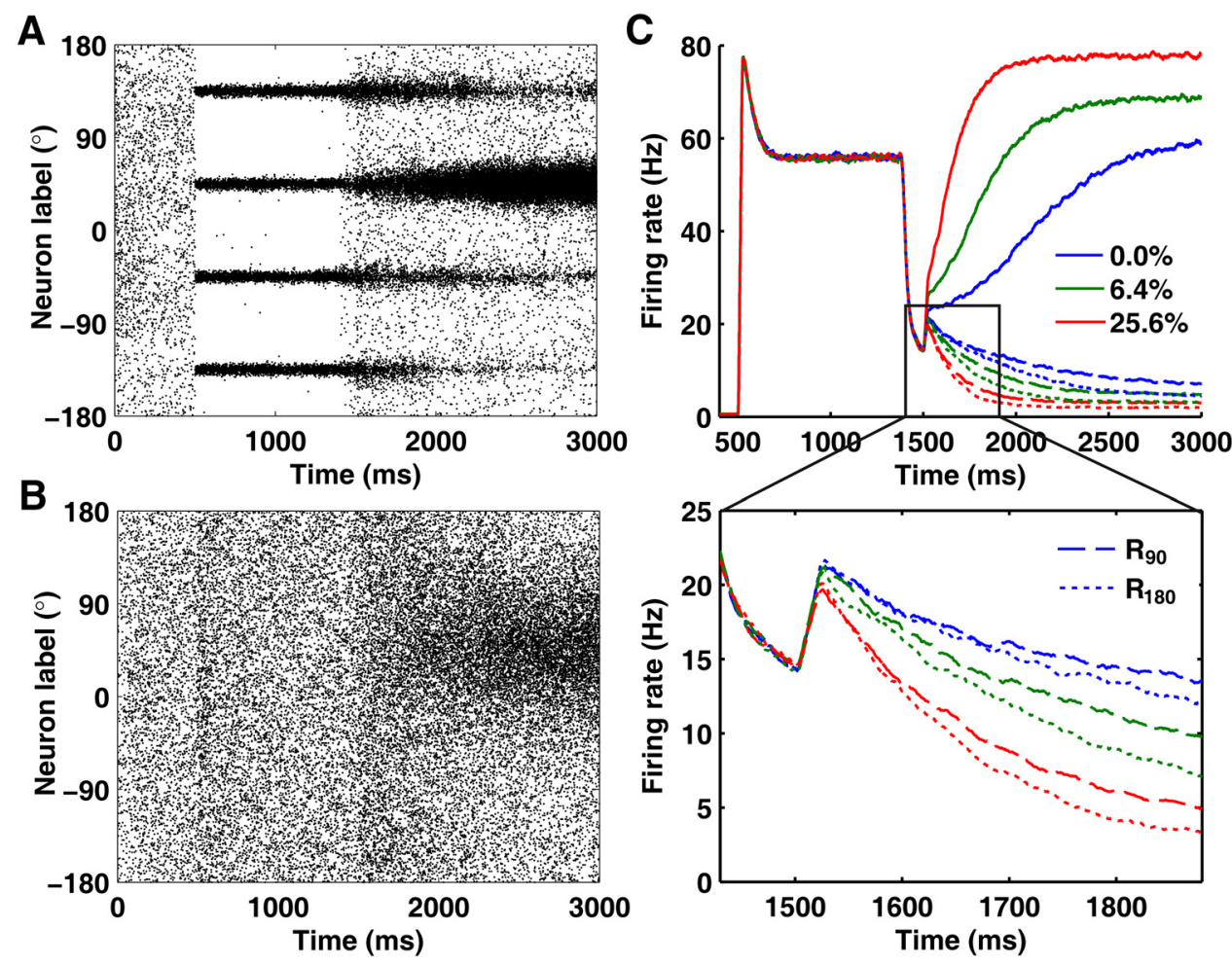

Figure 2. Neural activity during a four-choice motion-discrimination task at $J_{\text {sim }}=1.32$ and $J_{\text {opp }}=1.01 . \boldsymbol{A}, \boldsymbol{B}$, Spatiotemporal firing patterns of pyramidal cells $(\boldsymbol{A})$ and interneurons $(\boldsymbol{B})$ in a sample trial with $6.4 \%$ motion coherence. The coherent motion direction is at $45^{\circ}$. Each neuron is labeled by its preferred direction. $C$, Temporal evolution of mean firing rates of neural pools separately located at $0^{\circ}$ (solid), $90^{\circ}$ (dashed), and $180^{\circ}$ (dotted) relative to the coherent motion direction, averaged over 500 correct trials, for different coherence levels. A $0.45 \mathrm{~s} \mathrm{segment} \mathrm{is} \mathrm{expanded}$ at the bottom.

network dynamics and decision behaviors to parameter variations and found that the reported phenomena hold true when parameter values are within the physiological ranges. For example, the values of maximum recurrent synaptic conductance should be taken such that recurrent excitation is mainly mediated by NMDARs. For simplicity, here we did not highlight the differences in parameter values between the two models.

\section{Results}

There are a couple of modeling studies focused on the circuit mechanism and synaptic dynamics underlying random-dot motion perception. Especially, recurrent network models were constructed to model a local circuit in the lateral intraparietal area (LIP), which is considered to be a neural correlate of perceptual decision-making (Shadlen and Newsome, 2001; Roitman and Shadlen, 2002; Huk and Shadlen, 2005; Churchland et al., 2008). In those studies, the model network was composed of excitatory pyramidal cells and inhibitory interneurons (e.g., Furman and Wang, 2008; Liu and Wang, 2008). The pyramid-to-pyramid connectivity was heterogeneous, whereas the connectivity involving pyramidal cells and interneurons was homogeneous. In contrast, here we introduce inhomogeneous connectivity between interneurons and pyramidal cells to explore how structured inhibition can remarkably affect the network dynamics and decision behavior in a multiple-choice direction-discrimination task (Fig. 1A). Both types of neurons are directionally tuned, and their preferred directions separately cover a full circle uniformly (Fig. 1B). We assume that pyramidal cells receive two external input signals representing the visual targets and the motion stimulus; the temporal and spatial patterns of these inputs are depicted in Figure 1, $C$ and $D$, respectively. Compared with the study by Furman and Wang (2008), here we need not include either a normalization factor in the target input or a "topdown" control signal during motion viewing, because their effects on decision-making can arise naturally when structured synaptic inhibition is taken into account.

\section{Network dynamics and behavioral response}

The present model is able to account for the behavioral data and neuronal activity in LIP during motion discrimination. Figure $2 \mathrm{~A}$ shows the spatiotemporal firing pattern of pyramidal cells in a four-choice direction-discrimination task with motion coherence at $6.4 \%$. A trial begins when all cells are in a resting state, firing spontaneously at a few Hz. Four targets, with adjacent targets $90^{\circ}$ apart, are then displayed, indicating the four choice alternatives. The angular positions of targets are denoted by $\Theta_{i}$ $(i=1-4)$. After the appearance of the targets, four neural pools located around $\Theta_{i}$ are activated, leading to four symmetrical bumps in the profile of population activity. After the onset of the motion stimulus (at $1300 \mathrm{~ms}$ ), these neural pools decrease their activity transiently because of a reduced efficacy of target inputs, presumably representing a shift of attention from the targets to the random-dot display. The four neural pools then selectively integrate the stimulus input and compete against each other through feedback inhibition from interneurons. Finally, one neural pool wins the competition and increases its activity toward a decision threshold, whereas the activities of the others are essentially suppressed. Here, a decision is made in the form of a bell-shaped bump activity profile. Conversely, the activities of interneurons are nearly homogeneous before the stimulus input breaks the symmetry of network dynamics, and only those located around the direction of the selected target exhibit elevated 
A

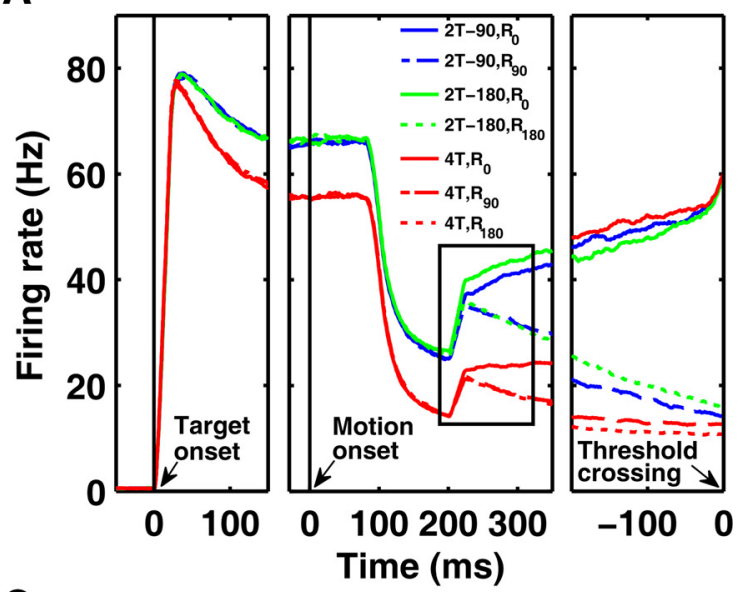

$\mathrm{C}$

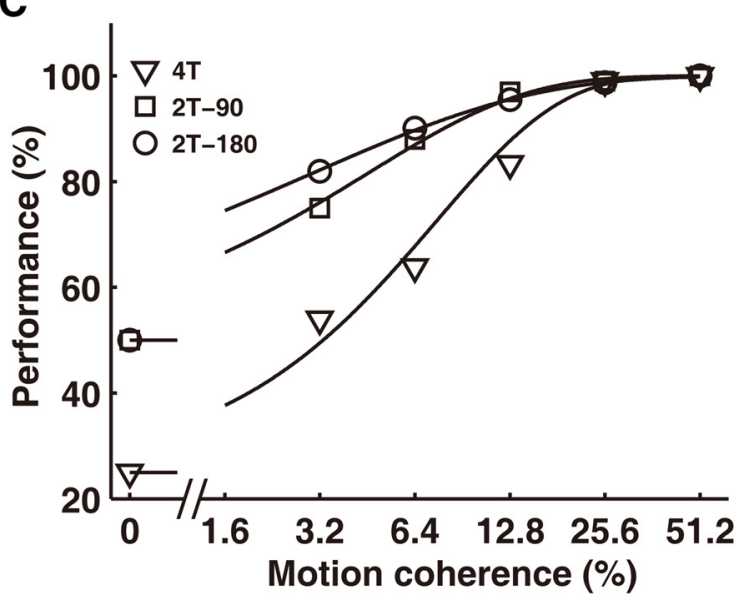

B

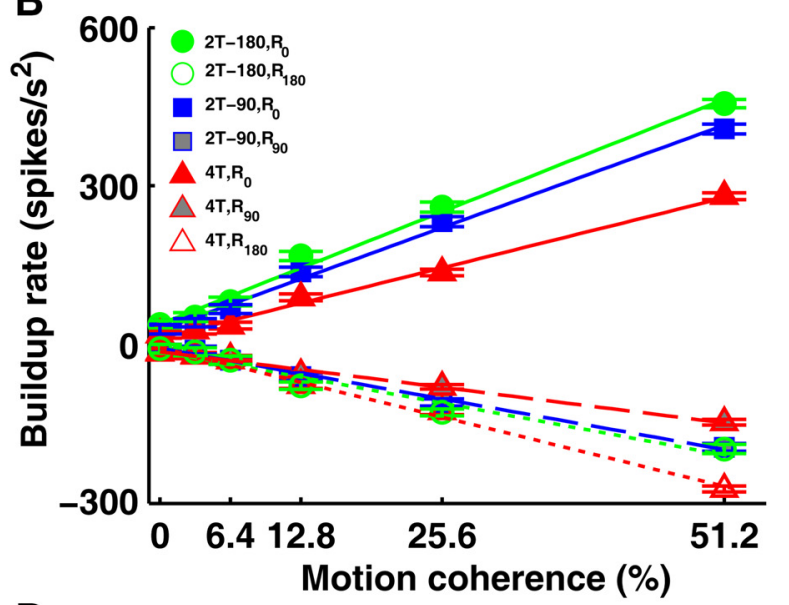

D

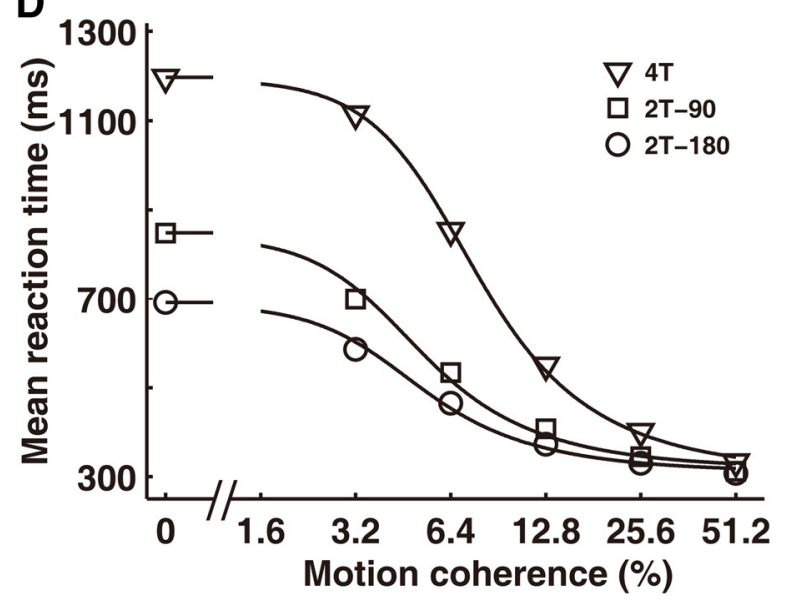

Figure 3. Comparison of neurophysiological and behavioral data between three conditions, in which two targets are separated by $180^{\circ}(2 \mathrm{~T}-180)$ or $90^{\circ}$ ( $\left.2 \mathrm{~T}-90\right)$, or four targets are evenly spaced (4T). $\boldsymbol{A}$, Temporal evolution of mean firing rates of neural pools located at $0^{\circ}$ (solid), $90^{\circ}$ (dashed), or $180^{\circ}$ (dotted) relative to the coherent motion direction in the $2 \mathrm{~T}-180$ (green), $2 \mathrm{~T}-90$ (blue), and $4 \mathrm{~T}$ (red) conditions. The temporal traces are aligned to target onset (left), motion onset (middle), and threshold crossing (right), respectively. The motion coherence is at $6.4 \%$. $\boldsymbol{B}$, Activity buildup rates, calculated over the time window of $190-320$ ms after motion onset (indicated by a rectangle in $A$ ), versus motion coherence. Each data point and error bar represent the average and SEM over 1000 correct trials. C, D, Percentage of correct trials ( $\boldsymbol{C}$ and mean reaction time on correct trials (D) versus motion coherence for the 2T-180 (circles), 2T-90 (squares), and 4T (downward triangles) conditions. A total of 1000 trials are counted for each case. $J_{\text {sim }}=1.32$ and $J_{\text {opp }}=1.01$ in all panels.

activity during decision formation (Fig. 2B). In the following, we focus on the activity of pyramidal cells.

The decision process can be revealed by showing time courses of mean firing rates of neural pools around each $\Theta_{i}$. Here, $R_{0}, R_{90}$, and $R_{180}$ denote the average firing rates of pyramidal cells whose preferred directions are along, orthogonal to, and opposite to, respectively, the coherent motion direction. In response to target presentation alone, $R_{0}, R_{90}$, and $R_{180}$ are nearly of the same values; they initially undergo a dramatic increase, followed by an adaptation to $\sim 56 \mathrm{~Hz}$ (Fig. 2C). After motion onset, they first decrease and then rise together in the early epoch. Such a dip may mark the initiation of evidence accumulation. On a correct trial, $R_{0}$ ramps up all the way, whereas $R_{90}$ and $R_{180}$ drop after some moment because of lateral inhibition, with $R_{180}$ decaying faster than $R_{90}$. A choice is made whenever a threshold level of $60 \mathrm{~Hz}$ is crossed first. Moreover, the buildup rate of $R_{0}$ during motion viewing rises with increasing the coherence level, and thus it takes less time to reach a decision. All these results agree well with experimental observations (Churchland et al., 2008).

We further compare neural responses in the two- and fourchoice tasks, in which two targets are separated by $180^{\circ}(2 \mathrm{~T}-180)$ or $90^{\circ}(2 \mathrm{~T}-90)$, or four targets are evenly spaced $(4 \mathrm{~T})$. Of note, firing rates are aligned to the target onset, motion onset, and crossing of decision threshold, respectively (Fig. 3A). There are three salient features worth noting. First, between target appearance and motion onset, there is a marked difference in firing rates between the two- and four-choice conditions. Compared with the $2 \mathrm{~T}-180$ task, firing rates are on average $11 \mathrm{~Hz}$ lower in the $4 \mathrm{~T}$ task and $1.4 \mathrm{~Hz}$ lower in the 2T-90 task. Second, the reduction in firing rates persists throughout the dip after motion onset. Third, the termination of decisions is accompanied by a crossing of fixed firing threshold under all conditions.

We also calculate the buildup rate of ramping activity over the epoch indicated by a rectangle in Figure $3 A$ on correct trials. The buildup rates scale linearly as a function of motion coherence (Fig. $3 B$ ). The buildup rate of $R_{0}$ rises with increasing the coherence level, whereas that of $R_{90}$ or $R_{180}$ drops. At a fixed coherence level, the buildup rate of $R_{0}$ falls with increasing the number of targets. In the four-choice task, there is a clear difference between the buildup rates of $R_{90}$ and $R_{180}$ at intermediate and high coherence levels, in contrast to a minor difference in the study by Furman and Wang (2008).

There exist marked differences in the behavioral data between the three conditions. Whereas the performance (i.e., percentage of correct trials) is similar in the $2 \mathrm{~T}-180$ and $2 \mathrm{~T}-90$ conditions (Fig. $3 C$ ), the mean reaction time (defined as the time required 
for the maximum firing rate to reach $60 \mathrm{~Hz}$ after motion onset) on correct trails is longer in the $2 \mathrm{~T}-90$ condition (Fig. $3 D$ ). In contrast, there is no such difference in reaction time in the study by Furman and Wang (2008). At low and intermediate coherence levels, the performance drops markedly and the reaction time rises remarkably in the $4 \mathrm{~T}$ condition compared with those in the $2 \mathrm{~T}-180$ condition. All the results shown in Figure 3 are consistent with the experimental observations (Churchland et al., 2008). As will be discussed in the following sections, structured inhibition from interneurons to pyramidal cells plays an essential role in realizing these features. Moreover, a speed-accuracy tradeoff, which was achieved by varying the control signal in the study by Furman and Wang (2008), can also be realized by modulating the strength of synaptic inhibition in our model.

\section{Opposite-feature inhibition and similarity effect}

In the four-choice task, $R_{90}$ has a lower decreasing rate than $R_{180}$ during decision formation on correct trials (Fig. $2 C$ ). This is an important feature, indicating that the neural activity not only encodes the information about the choice itself but also reflects the likelihood of a decision to be correct, as suggested by theoretical studies (Ma et al., 2006; Beck et al., 2008). This also implies that the more similarity two neural pools share in their preferred directions, the more correlated their activities become. This similarity effect mainly stems from opposite-feature inhibition, which leads to stronger lateral inhibition between pyramidal cells with larger angular distance. Consequently, two excitatory neural pools with opposite preferred directions tend to repress each other remarkably, which intensifies the competition between them. Thus, their firing rates, like $R_{0}$ and $R_{180}$, are most anticorrelated during decision-making. As the winning neural pool builds up its activity, the neural pool with opposite preferred directions has the lowest buildup rate. Remarkably, the buildup rate of $R_{0}$ scales linearly as a function of motion coherence and rises with increasing $J_{\text {opp }}$ (determining the connectivity strength of opposite-feature inhibition; Fig. $4 A$ ). Moreover, the buildup rate of $R_{90}$ is higher than that of $R_{180}$, and this difference is significantly enlarged with greater $J_{\text {opp }}$ (Fig. $4 B$ ), consistent with the experimental findings (Churchland et al., 2008). There is no such difference if recurrent inhibition is homogenous (Furman and Wang, 2008).

Another aspect of the similarity effect was once predicted by Furman and Wang (2008): in an eight-choice task with intermediate motion coherence, the probability of making an erroneous choice toward a target adjacent to the correct one was the highest compared with choosing other targets. This feature is also present in our data (Fig. 4C); furthermore, the probability of selecting one incorrect target decays continuously with less similarity between that and the correct target. Because the motion stimulus biases the choice toward the correct target, stronger inhibition is exerted on neurons located opposite to or far away from the coherent motion direction. Therefore, it is more difficult for these neurons to win the competition. Moreover, increasing $J_{\text {opp }}$ markedly decreases the possibility of choosing the opposite target relative to the correct one.

\section{Elimination process}

In behavioral science, it is well known that, when required to select an item from multiple alternatives, subjects tend to first eliminate the least likely ones to reduce the choice set (Tversky, 1972; Yaniv and Schul, 1997; Roe et al., 2001), known as elimination process. Such a strategy can speed up the decision process because, during evidence accumulation, the input information
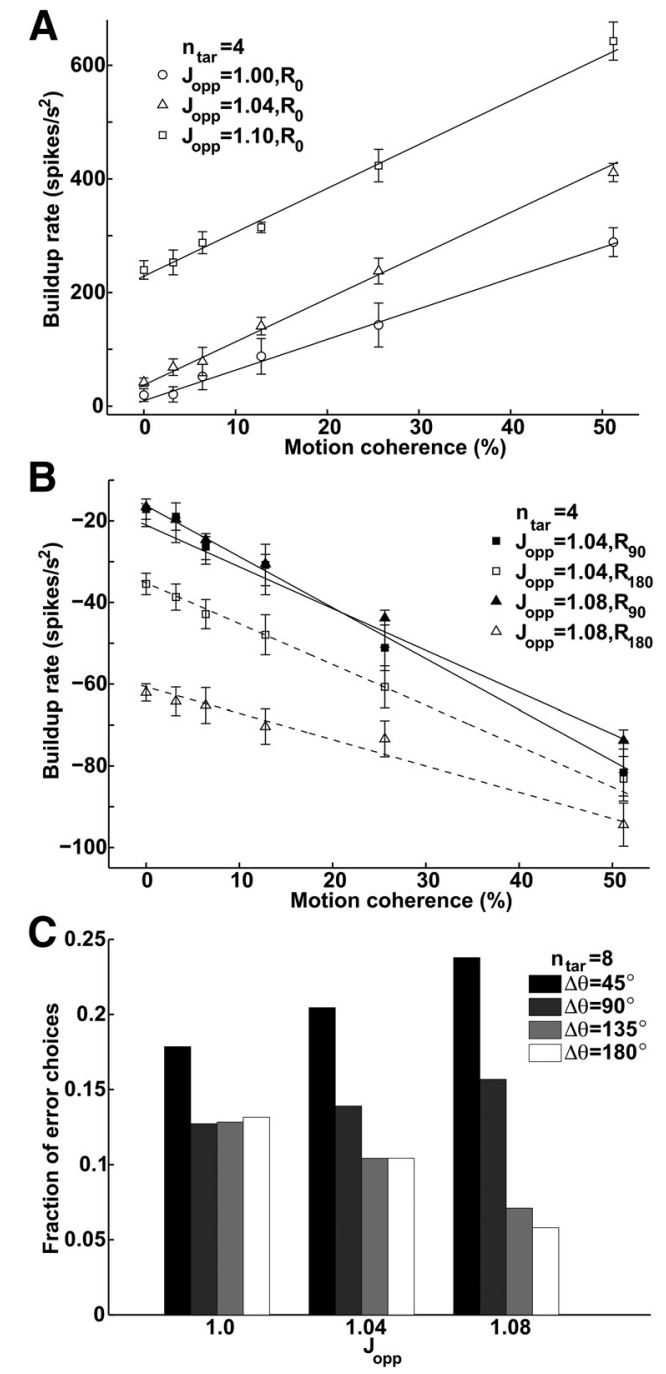

Figure 4. Effects of opposite-feature inhibition on firing activity of pyramidal cells and decision-making. $A$, Buildup rate of neural activity around the direction of the correct target in a four-choice task versus motion coherence. At the same coherence level, the buildup rate rises with increasing $J_{\text {opp }}$ B, Buildup rates of neural activity around $90^{\circ}$ (solid) or $180^{\circ}$ (dashed) relative to the direction of the correct target versus motion coherence. The difference between the two cases is enlarged with increasing $J_{\text {opp }}$. Each data point and error bar in $\boldsymbol{A}$ and $\boldsymbol{B}$ represent the average and SEM over 1000 correct trials, calculated over the period of $190-320 \mathrm{~ms}$ after motion onset. C, Spatial distribution of error choices in an eight-choice task with 3.2\% coherence. The histograms display the probability of choosing a target at different angular distance $\Delta \theta$ from the correct one for different values of $J_{\text {opp }} . J_{\text {sim }}$ is fixed at 1.1 in all panels.

can be used not only in favor of one option but also against others. We find that the elimination process also occurs in multiple-choice motion-discrimination tasks and can be considered a natural extension of the similarity effect induced by opposite-feature inhibition. That is, the targets that have a greater angular difference from the chosen target are ruled out of the choice set earlier. As an operational definition, we call the "elimination" of a choice alternative when the neural representation of this alternative falls below one-third of the highest firing rate at that time. This is a practical definition: despite the stochasticity in external input, a neural pool is generally unlikely to win the competition when its activity is much lower compared with other active ones.

To demonstrate the dynamic feature that may underlie such a decision strategy, we simulate the neural activity in an eightchoice motion-discrimination task. Notably, the neural pools lo- 

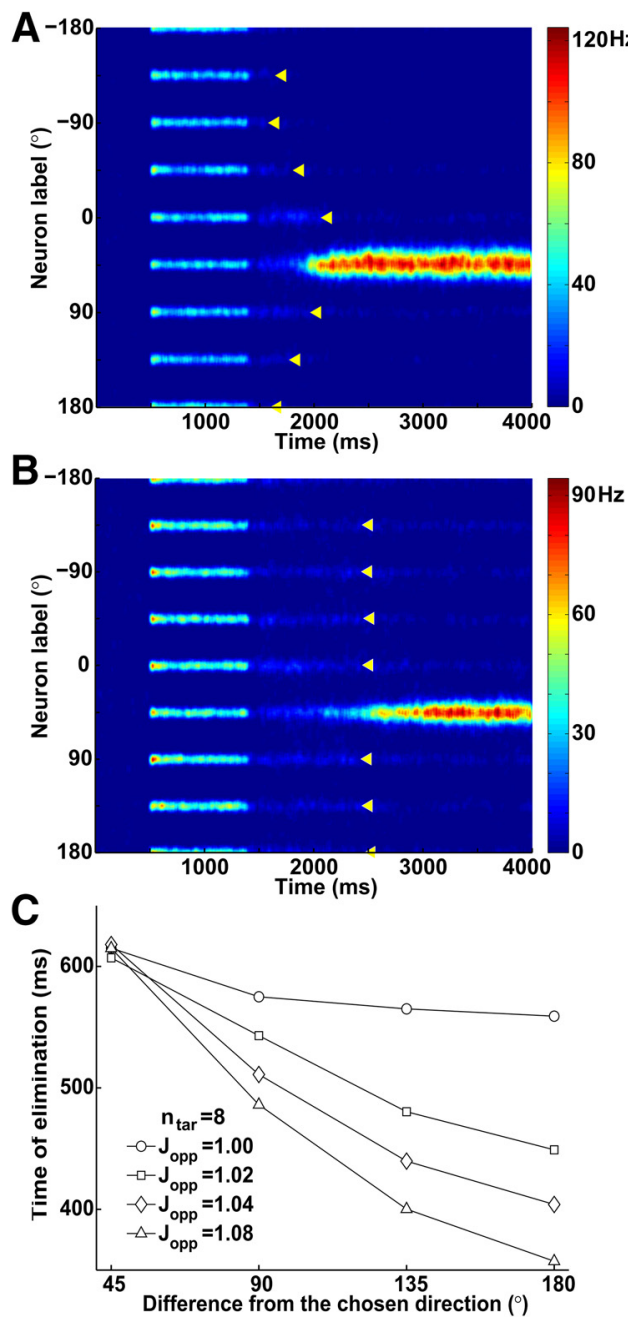

Figure 5. Elimination effect in an eight-choice task with $6.4 \%$ coherence. Color-coded spatiotemporal firing pattern of pyramidal cells at $J_{\text {opp }}=1.06(\boldsymbol{A})$ or $J_{\text {opp }}=1.00(B)$. Yellow arrows indicate the elimination time for the seven distracting choices. An option is considered to be removed from the choice set when the firing rate of neurons around the direction of this target falls below one-third of the maximum firing rate at the same time. $C$, The mean time required to eliminate one option relative to the stimulus onset, averaged over 500 correct trials, versus its angular distance from the correct target for different values of $J_{\text {opp. }} J_{\text {sim }}$ is kept at 1.1 in all panels.

cated more distant from the direction of the chosen target first decrease their activity remarkably (Fig. 5A). The neural pools around $\Theta_{i}$ (except the winning pool) lose the competition one by one, and the corresponding options can be considered to be removed from the choice set. Such an elimination effect depends remarkably on $J_{\mathrm{opp}}$. If $J_{\mathrm{opp}}=1.0$, no option is ruled out significantly ahead of others, especially for those at $\Delta \Theta=90^{\circ}, 135^{\circ}$, or $180^{\circ}$ relative to the direction of the selected target (Fig. $5 B$ ). Thus, the elimination effect is mostly associated with opposite-feature inhibition. Indeed, the elimination effect becomes more pronounced when $J_{\text {opp }}$ is increased, especially for greater $\Delta \Theta$ (Fig. $5 C)$. This prediction awaits experimental justification.

Consistent with psychological studies (Roe et al., 2001) and our daily experience, an apparent advantage of the elimination process is that, by ruling out unlikely options and leaving only few promising candidates for inspection, the multiple-choice discrimination task becomes much easier. Thus, the reaction time decreases markedly with increasing $J_{\text {opp }}$, especially at lower coherence levels (Fig. 6A). Here, opposite-feature inhibition con-
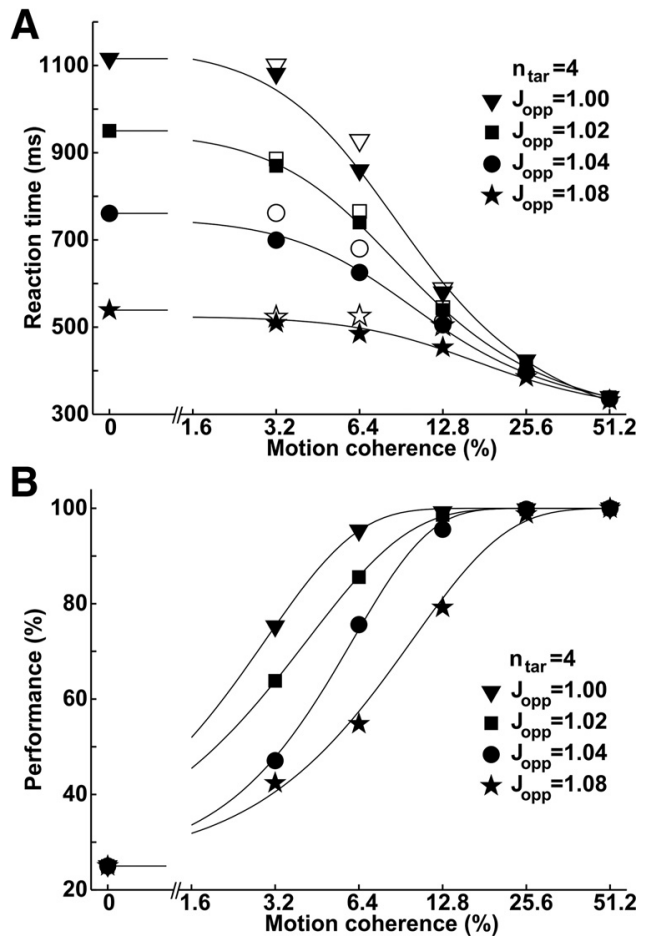

Figure 6. Effect of opposite-feature inhibition on decision behavior in a four-choice task. $\boldsymbol{A}$ Mean reaction time versus motion coherence on correct (filled symbols) and error (open symbols) trials. $\boldsymbol{B}$, Percentage of correct trials as a function of motion coherence. A total of 1000 trials are counted for each case. The performance decreases with increasing $J_{\text {opp }}$ at low and intermediate coherence levels, whereas the reaction time drops with increasing $J_{\text {opp }} . J_{\text {sim }}$ is fixed at 1.1.

tributes remarkably to neural competition. Because the neural pools with opposing directional preferences tend to inhibit each other, random fluctuations in input can easily upset the balance between them: the ramping-up activity of one neural pool will suppress the activity of the other pool, and this in turn disinhibits itself. On the other hand, strong opposite-feature inhibition may cause hasty decisions; for example, random fluctuations in the motion stimulus input may lead to an early elimination of the correct option. Thus, the percentage of correct trials drops with increasing $J_{\text {opp }}$ at low and intermediate coherence levels (Fig. 6B). That is, a quick decision may be made at the expense of decision accuracy.

\section{Similar-feature inhibition and fine discrimination}

Experimental studies indicate that many species are able to make categorical choices among very similar options, namely capable of fine discrimination (De Bruyn and Orban, 1988; Hol and Treue, 2001; Purushothaman and Bradley, 2005; Jazayeri and Movshon, 2007). However, most of the continuous network models failed to reproduce the feature of fine discrimination. For example, adjacent bumps of activity merge on half of the simulation trials, preventing the circuit from making a choice in an eight-choice task (Furman and Wang, 2008). If two targets are separated by $30^{\circ}$ in a two-choice task, our model with only opposite-feature inhibition also fails to distinguish between the two targets (Fig. 7A). Instead, similar-feature inhibition has a critical role in fine discrimination. With $J_{\text {sim }}=1.4\left(J_{\text {sim }}\right.$ determining the connectivity strength of similar-feature inhibition), a single bump of activity profile can emerge around the direction of one target (Fig. $7 B$ ). In fact, there exists a well defined threshold of angular distance between two targets, above which most trials 

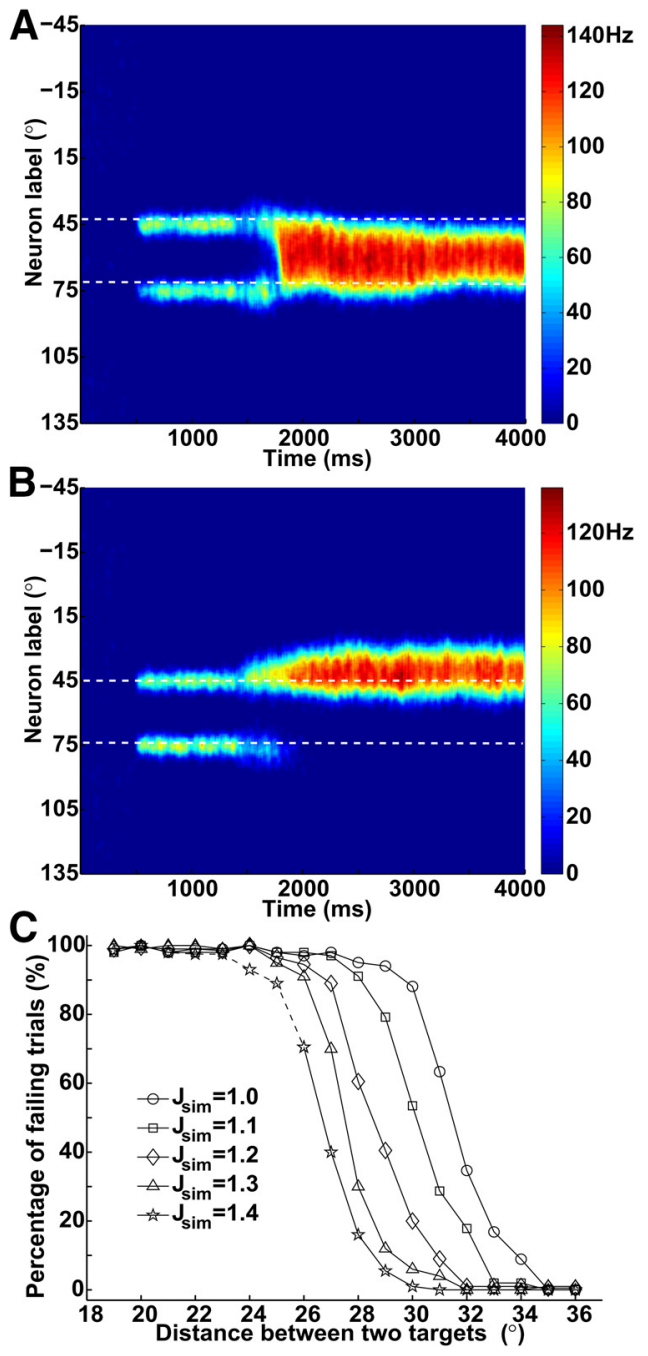

Figure 7. Fine discrimination in a two-choice task with $0 \%$ coherence. Color-coded activity of pyramidal cells in a task with two targets separated by $30^{\circ}$ at $J_{\text {sim }}=1.0(\boldsymbol{A})$ or $J_{\text {sim }}=1.4(\boldsymbol{B})$. C, Percentage offailing trials versus the angular difference between the two targets for different values of $J_{\text {sim. }}$. A total of 1000 trials are counted in each case. The dashed part of the curve indicates that, because of strong local inhibition, the network is in the spontaneous state on some trials. These trials are excluded from calculating the percentage. $J_{\text {opp }}$ is kept at 1.05 in all panels.

display a winner-take-all competition and below which most trials end up with merging of activity bumps around the directions of two targets (Fig. 7C). This threshold shifts leftward with increasing $J_{\text {sim }}$, indicating an improvement in the capability of fine discrimination. Together, similar-feature inhibition may serve as an effective mechanism to enhance fine discrimination.

To explore why similar-feature inhibition can facilitate fine discrimination, we reduce the spiking network model to a simplified ring model (see Materials and Method). In this model, although the information about temporal integration of sensory evidence to the final steady state is lost, the steady states and their local stability can be determined without running thousands of kinetic simulations. By finding the stable steady states of the simplified model, we identify all the possible outcomes in the spiking network model; in contrast, the unstable steady states cannot be maintained over time in the spiking network model, because the network state will be driven away from them as a result of stochastic fluctuations in both the stimulus input and time delays in synaptic transmission.
If two targets are sufficiently different in a two-choice task, there appear two stable steady states with their peak activity at the direction of either target, together with one unstable steady state with its peak activity intermediate between the directions of the two targets (Fig. 8A, top). Thus, the network dynamics will be driven to either of two stable states even at $0 \%$ coherence, and a choice is made between the two targets. When decreasing the angular difference between two targets past some threshold, however, the network dynamics undergo a saddle-node bifurcation. Consequently, the two stable steady states vanish, and there appears only one stable steady state with the peak activity in between the directions of the two targets (Fig. 8A, bottom). Thus, the network dynamics will always be attracted to this state regardless of decision-making, without differentiation between the two targets. Of note, the stability of the steady state with its peak activity located between the directions of two targets can be characterized by calculating the maximum eigenvalue $\lambda_{m}$ of the Jacobian matrix (see Eq. 29). As expected, $\lambda_{m}$ goes from positive to negative values with decreasing the angular distance $\Delta \theta$ between two targets (Fig. $8 B$ ). Moreover, the bifurcation point, at which $\lambda_{m}=0$, shifts to smaller $\Delta \Theta$ values with increasing $J_{\text {sim }}$; that is, the least discriminable difference between two targets gets smaller with greater $J_{\text {sim }}$. Collectively, the similar-feature inhibition contributes remarkably to fine discrimination.

To understand what happens at the saddle-node bifurcation point and why it is affected much by similar-feature inhibition, we need to note two features. First, when two targets get close, they also activate neurons located between the directions of the two targets. This excitation term in the input acts like an "attracting force" between the two bumps of activity profile, which tends to induce a single, wider bump. Second, the representation of one motion direction suppresses that of similar motion directions (Neri and Levi, 2009). When two targets become similar, the feedback inhibition gets stronger for neurons located between the directions of the two targets. The above features are illustrated in Figure $8 C$. At the early stage of temporal integration of sensory evidence, the elevated pyramidal activities elicit a wide bump of activity profile in interneurons. Consequently, the pyramidal cells located between the directions of the two targets are inhibited strongly. This inhibition acts as a "barrier," preventing two bumps of activity from merging. At the saddle-node bifurcation point, the attracting force is marginally/critically strong enough to overcome the barrier; after two targets get any closer, merging of adjacent bumps dominates the network dynamics. Moreover, increasing $J_{\text {sim }}$ improves the inhibitory barrier. Therefore, the saddle-node bifurcation occurs at smaller angular differences with greater $J_{\text {sim }}$.

\section{A deliberate and more time-consuming decision strategy}

Besides fine discrimination, similar-feature inhibition also brings other dynamic effects to the decision-making circuit. Compared with opposite-feature inhibition, the similar-feature inhibition exerts a more negative control over neural activity. Any temporary fluctuation in pyramidal activity will be followed by a change in the activity of interneurons with similar preferred directions. The feedback inhibition attributable to similar-feature inhibition tends to counteract fluctuations in pyramidal activity. This naturally leads to two effects on the decision process. On one hand, it holds back the buildup of neural activity during evidence accumulation, and thus the reaction time rises with increasing $J_{\text {sim }}$ at 

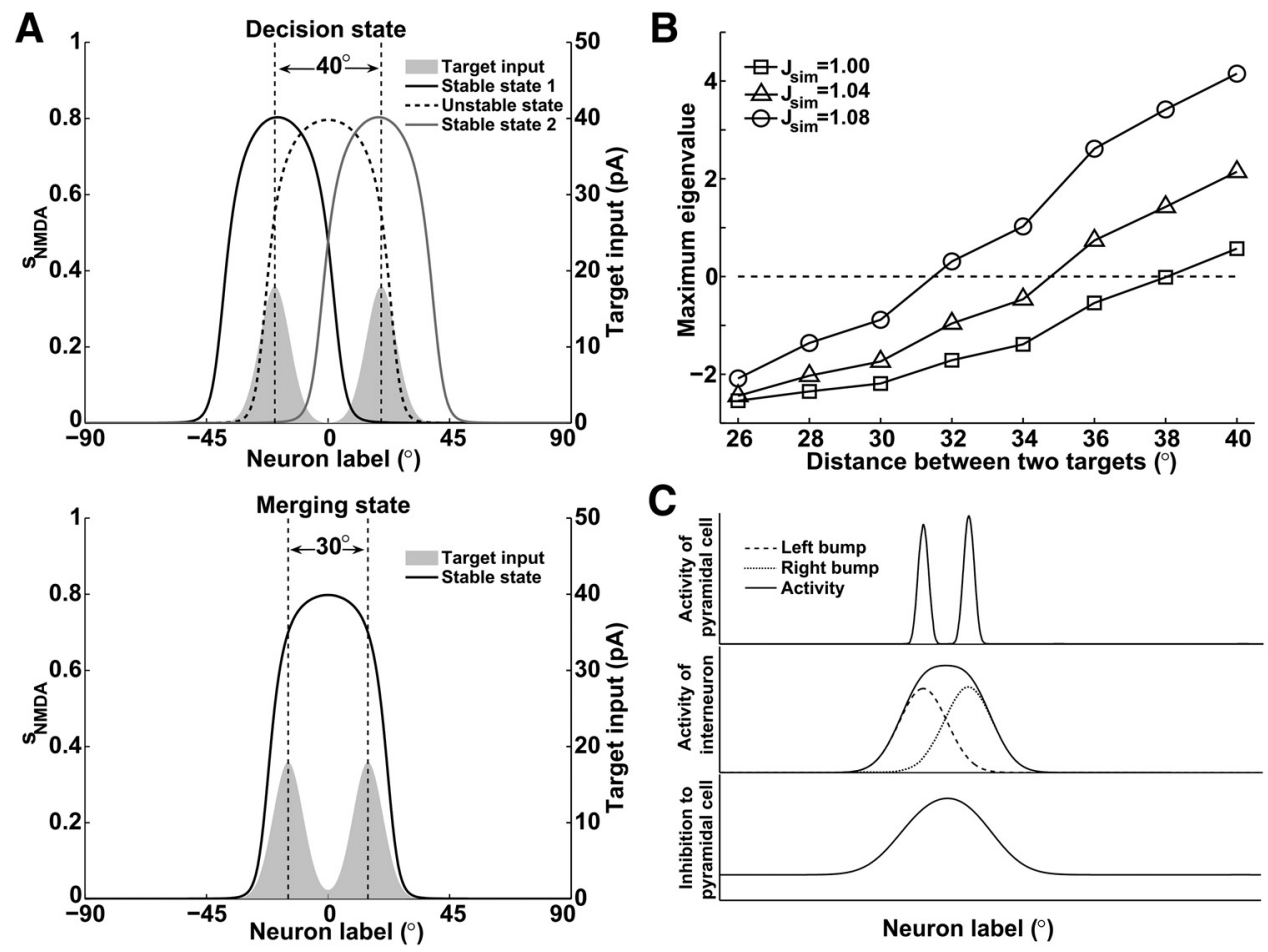

Figure 8. Illustration of fine discrimination in a two-choice task with $0 \%$ coherence using a simplified model. $\boldsymbol{A}$, In the decision state (e.g., when 2 targets are separated by $\left.40^{\circ}\right)$, the network has two stable steady states with peak activity (represented by $\mathrm{S}_{\mathrm{NMDA}}$ ) at the direction of either target and an unstable steady state. In the merging state (e.g., when 2 targets are separated by $30^{\circ}$ ), the merging of bumps at the direction of two targets constitutes the only stable steady state. $\boldsymbol{B}$, Maximum eigenvalue of the Jacobian matrix for the steady state with its peak activity located between the directions of two targets versus the angular distance between the two targets. A merging state occurs when the maximum eigenvalue is positive. The curve shifts leftward with increasing $J_{\text {sim. }}$. $J_{\text {opp }}$ is fixed at 1.05. C, Schematics of neural firing activity. The activity of pyramidal cells induced by two targets further elicits a bump of activity profile in interneurons, which peaks between the directions of two targets. The similar-feature inhibition exerts stronger inhibition to pyramidal cells located between the directions of the two targets, preventing the two bumps from merging.
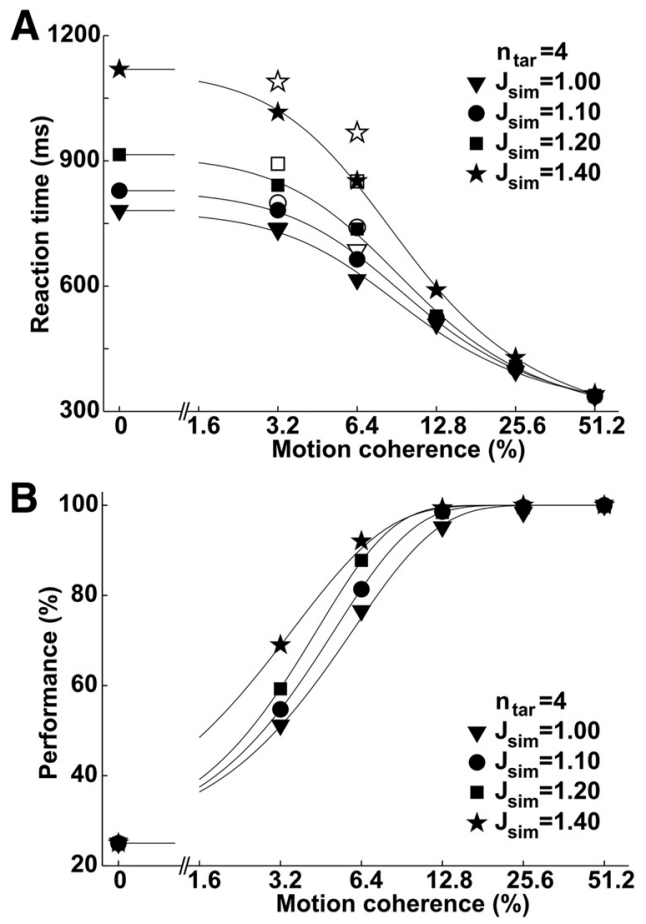

Figure 9. Effect of similar-feature inhibition on decision behaviors. Mean reaction time on correct (filled symbols) and error (open symbols) trials $(\boldsymbol{A})$ and percentage of correct trials $(\boldsymbol{B})$ in a four-choice task versus motion coherence for different values of $J_{\text {sim. }}$. A total of 1000 trials are counted in each case. $J_{\text {opp }}$ is fixed at 1.05 in all panels. low coherence levels (Fig. 9A). On the other hand, the process of evidence accumulation is more robust against random fluctuations, and thus the decision accuracy improves with increasing $J_{\text {sim }}$ (Fig. 9B).

With inclusion of similar-feature inhibition, the current model has several advantages over the model of Furman and Wang (2008) in accounting for the experimental observations in the study by Churchland et al. (2008) as shown above. Here, we make an additional discussion on the results shown in Figure 3. As adjacent targets become more similar, mutual inhibition between the corresponding neural pools grows stronger (e.g., the $2 \mathrm{~T}-90$ vs the $2 \mathrm{~T}-180$ condition). This effect is even stronger in the $4 \mathrm{~T}$ condition, because the similar-feature inhibition has a role from both sides of the feature dimension. Moreover, target inputs are increased in the $4 \mathrm{~T}$ case and feedback inhibition is generally enhanced. Consequently, neural pools around the direction of each target are laterally inhibited more strongly in the $4 \mathrm{~T}$ condition compared with the $2 \mathrm{~T}-90$ and $2 \mathrm{~T}-180$ conditions, and there is a clear difference in neural activity between them during the premotion period.

After the dip following the motion onset, firing rates ramp up for neural pools around the direction of each target, and thus lateral inhibition between them is improved to slow down the buildup of activity. This effect is enhanced when adjacent targets get closer (e.g., the $2 \mathrm{~T}-90$ vs the $2 \mathrm{~T}-180$ condition) and is even stronger in the $4 \mathrm{~T}$ condition. Thus, the decision process in the $2 \mathrm{~T}-90$ condition is slower than in the $2 \mathrm{~T}-180$ condition yet faster than in the $4 \mathrm{~T}$ condition. Together, the similar-feature inhibition has an essential role in the two- and four-choice direction-discrimination tasks. 

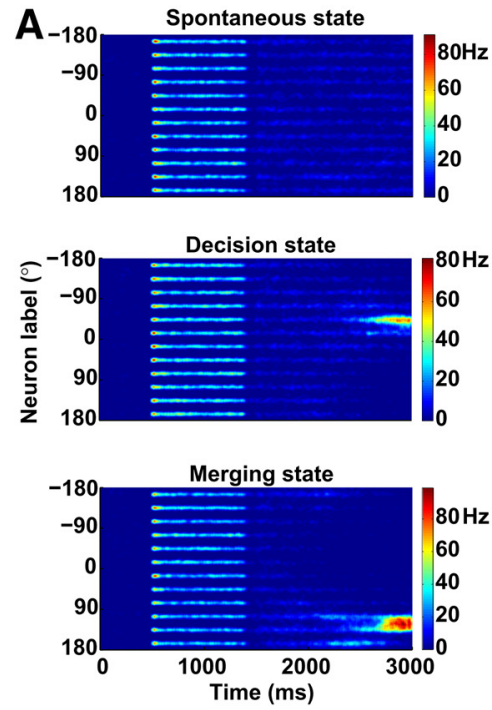
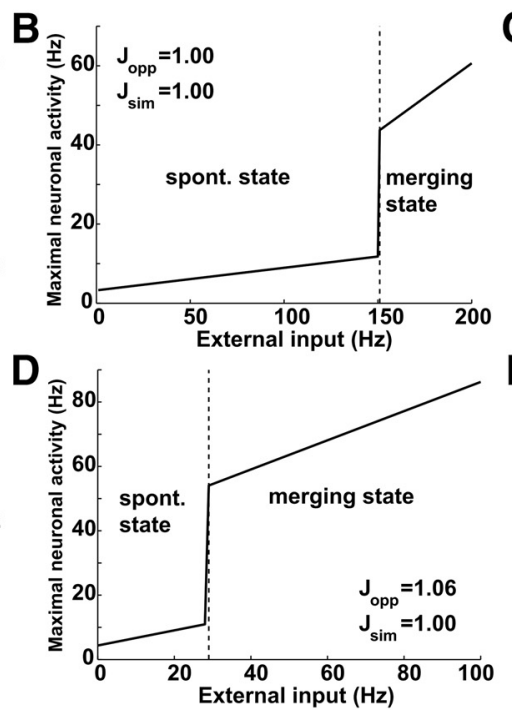
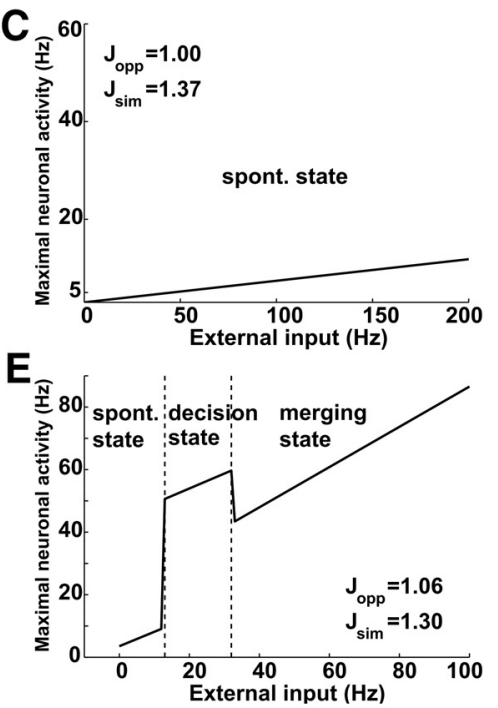

Figure 10. Neural activity and decision behavior in a 12-choice task with $0 \%$ coherence. $A$, Sample trials ending in a spontaneous state $\left(J_{\text {opp }}=1.00\right.$ and $\left.J_{\text {sim }}=1.37\right)$, decision state $\left(J_{\text {opp }}=1.06\right.$ and $\left.J_{\text {sim }}=1.30\right)$, or merging state $\left(J_{\text {opp }}=1.06\right.$ and $\left.J_{\text {sim }}=1.00\right)$. $\boldsymbol{B}-\boldsymbol{E}$, Maximum firing rate of pyramidal cells versus the external input. $\boldsymbol{B}$, With homogeneous inhibition $\left(J_{\text {opp }}=1.00\right.$ and $J_{\text {sim }}=$ $1.00)$, the network state converts directly from a spontaneous state to a merging state as the external input rises. $C$, With similar-feature inhibition only $\left(J_{\text {opp }}=1.00\right.$ and $\left.J_{\text {sim }}=1.37\right)$, the spontaneous state is too stable to be broken by physiologically reasonable inputs. $D$, With opposite-feature inhibition only $\left(J_{\text {opp }}=1.06\right.$ and $\left.J_{\text {sim }}=1.00\right)$, the spontaneous state is easier to break, yet the merging state is still more stable than the decision state. $\boldsymbol{E}$, With both opposite-feature and similar-feature inhibition $\left(J_{\text {opp }}=1.06\right.$ and $\left.J_{\text {sim }}=1.30\right)$, there is a region of external input in which the decision state is more stable than the other two states.

\section{Coordination between opposite-feature and similar-feature inhibition expands the range of decision-making}

Apparently, the opposite-feature inhibition and similar-feature inhibition have contrasting effects on various aspects of decisionmaking. For instance, the similar-feature inhibition tends to slow down the attractor dynamics, whereas the opposite-feature inhibition tends to speed up the decision process. Nevertheless, this also raises the possibility that they can cooperatively contribute to cognitive functions by complementing each other under certain conditions. Here, we propose a possible function originating from the combined inhibition, which cannot work via either of them.

When faced with an extremely difficult multiple-choice task, the network may be in one of three states: (1) none of the neural pools wins and all pools are in a low firing state (spontaneous state); (2) one neural pool wins the competition and a categorical choice is made (decision state); or (3) at least two neural pools have elevated firing activities and no categorical choice can be made (merging state). Figure $10 \mathrm{~A}$ shows three sample trials in a 12 -choice task with $0 \%$ coherence, separately corresponding to the above three cases. Clearly, only the decision state is desirable for a decision-making circuit. The range of external inputs, over which the circuit ends up in a decision state, is referred to as the "range of decision making" (Albantakis and Deco, 2009). The external input less than the lower limit of this range mostly leads to a spontaneous state, whereas the input greater than the upper limit mostly results in a merging state.

We further use the simplified ring model to analyze neural activity in the 12 -choice task with $0 \%$ coherence. We examine the ranges of external input over which the circuit separately ends up in a spontaneous, decision, or merging state. We then compare the differences in these ranges when the circuit is endowed with (1) homogeneous inhibition, (2) similar-feature inhibition only, (3) opposite-feature inhibition only, or (4) both opposite-feature and similar-feature inhibition. In such a difficult task as to picking 1 of 12 choice alternatives, it is a challenge for the decision circuit to guarantee the existence and uniqueness of the solution.
Given homogeneous inhibition, the spontaneous state is very stable, and the symmetry involved cannot be broken until a very strong input is applied (Fig. 10B). With similar-feature inhibition alone, the spontaneous state becomes even more stable against random fluctuations, to such an extent that the decision threshold cannot be reached by the activity of neurons around the direction of any target (Fig. 10C). With opposite-feature inhibition only, the network has difficulty in making a categorical choice even when it receives a strong input, because the emerging bump attractor tends to mix up the activities of adjacent neural pools (Fig. 10D). Thus, a range of decision state is possible only when both opposite-feature and similar-feature inhibition are present with proper strength (Fig. 10E). Notably, opposite-feature and similar-feature inhibition have different roles in a multiplechoice discrimination task: the former contributes to the initial breaking of the symmetric spontaneous state, whereas the latter reinforces the winner-take-all competition. They can function in a coordinated manner and integrate their advantages into a single circuit. All these results await experimental justification.

This coordination between opposite-feature and similarfeature inhibition stems from their distinct ranges of action. As experimental studies suggested (Rao et al., 1999), the oppositefeature inhibition functions between macro-columns and affects neurons over a wide range of preferred directions $\left(120^{\circ}-180^{\circ}\right)$. In contrast, the similar-feature inhibition operates between nearby micro-columns (or mini-columns) (Kaas, 2012). In our model, the similar-feature inhibition affects a relatively small population of neurons with similar feature preference (see Eq. 12). Accordingly, the similar-feature inhibition does not have a significant effect on the competition between adjacent neural pools until their activities rise to sufficiently high levels. Thus, at the beginning of a decision process, when all neurons fire at low rates, the elimination effect attributable to opposite-feature inhibition dominates evidence accumulation; in the later phase, when only few options are left for competition, the fine-discrimination effect attributable to similar-feature inhibition plays a role and warrants the uniqueness of decision-making. Together, the dif- 
ference in the range of action between the two inhibitory structures leads to two separate temporal stages for their functioning, which allows a cooperative effect of the seemingly counteracting structures.

\section{Discussion}

In this study, we demonstrated how the structure of interneuronto-pyramid connectivity sensitively affects the attractor dynamics and decision behavior in a decision-making circuit. The broad opposite-feature inhibition endows the circuit with an elimination strategy, speeding up the decision process at the cost of decision accuracy; the narrow similar-feature inhibition significantly enhances the capability of fine discrimination of the circuit and improves the decision accuracy, while tending to slow down the decision process. The combination of both structures with proper strength enhances the ability of the circuit to reach a decision state when faced with a multiple-choice motiondiscrimination task. The model successfully accounted for experimental observations and made experimentally testable predictions.

\section{Justification of opposite-feature inhibition}

Although there are few studies on opposite-feature inhibition in decision-related areas such as LIP, it has been extensively observed in other areas. (1) For example, in response to static bar stimuli, stronger inhibition exists between V1 columns with orthogonal preferred orientations, suggesting stronger lateral inhibition between columns with opposite preferred features (Morrone et al., 1982; Wörgötter and Koch, 1991; Brouwer and Heeger, 2011). (2) The columnar structure in area MT is organized such that, along one tangential direction of the cortex, the preferred direction of each column changes gradually, whereas along another tangential direction of the cortex, adjacent columns have opposite preferred directions. This topographically lays ground for the coexistence of similar-feature and oppositefeature inhibition (Albright et al., 1984). (3) The cells in the prefrontal cortex show lower responses to antipreferred stimuli than other nonpreferred stimuli (Funahashi et al., 1989; Rao et al., 1999; Krimer and Goldman-Rakic, 2001), suggesting the presence of lateral inhibition between neurons with opposite-feature preference. Given the presence of opposite-feature inhibition across different brain regions of various species, chances are high that it also exists in perceptual decision-making-related regions.

\section{Hasty decisions associated with opposite-feature inhibition}

We demonstrated that opposite-feature inhibition is closely associated with the elimination process, speeding up the decision process at the expense of decision accuracy. In previous models, homogeneous feedback inhibition contains no information about the directional bias (Furman and Wang, 2008). However, with opposite-feature inhibition, the information encoded in the sensory input is integrated as evidence not only in support of some choice alternative but also against others. This undoubtedly accelerates the decision-making, while rendering the decision outcome more vulnerable to random fluctuations in inputs in the early phase of sensorimotor integration.

Moreover, the opposite-feature inhibition has a role in breaking the symmetry of network dynamics, and the decision process will be accelerated if choice targets are spatially asymmetric and more similar (e.g., in the 2T-90 condition). If the connectivity strength of opposite-feature inhibition is relatively strong, the mean reaction time could be shorter on 2T-90 trials than on 2T-180 trials, in contrast to the experimental data (Churchland et al., 2008). This is why a relatively small value of $J_{\text {opp }}$ is taken when simulating the two- and four-choice tasks with a common parameter set (Fig. 3).

\section{Two phases of a decision-making process}

We propose that the multiple-choice decision-making process can be approximately divided into two phases: (1) the coarse selection; and (2) fine selection. Notably, opposite-feature inhibition operates on a much wider range than similar-feature inhibition in our model. After motion onset, the activities of neural pools located around the direction of each target build up over time, and the opposite-feature inhibition predominates the network dynamics. The information encoded in the stimulus input is mainly used to screen out unfavorable options one by one, until few adjacent alternatives are to be differentiated. Then, the similar-feature inhibition begins to have a role. This narrow inhibition operates locally to ensure that the bump of activity profile appears only around the direction of one target. The relative strength of opposite-feature versus similar-feature inhibition affects the transition between the two phases.

One experimental design to justify the two-phase hypothesis could be as follows. In a normal eight-alternative motiondiscrimination task, the coherent motion direction in the display is changed randomly at any time. At the same time, a change in the color of the fixation point informs the monkey that the motion direction has changed. The monkey is rewarded provided that it reports either the original or the current motion direction. Clearly, both the timing and magnitude of directional changes affect the decision performance and reaction time, and their different combinations should influence the decision behavior to various degrees given the presence of two phases in decisionmaking. One prediction is as follows. Among trial blocks in which the directional changes occur at the same time and the monkey correctly reports the current motion direction, the mean reaction time is longer on trials with larger magnitudes of directional changes.

\section{A comparison with previous models}

Since the publication of Churchland et al. (2008), several attempts have been made to account for experimental observations (Beck et al., 2008; Furman and Wang 2008; Albantakis and Deco 2009). Our model is an extension of the Furman and Wang model. With the inclusion of structured inhibition, we reproduced both the physiological and behavioral data from the monkey experiment, without making additional assumptions about target inputs and control signal as done by Furman and Wang (2008). Of note, even without a normalization factor in target input depending on the number of targets, the firing rates during the premotion period would still drop, to different extents, when increasing the number of targets in the Furman and Wang model. This is realized through a global normalization substantiated by unspecific inhibition from interneurons. When the number of targets rises, homogenous feedback inhibition from interneurons rises because of increased target inputs. The reason why an additional normalization factor is included in target input could be that the internal global normalization alone is insufficient to induce a similar drop in firing rates to the experimental data. In contrast, similar-feature inhibition provides a stronger "local normalization" mechanism: an increase in choice number induces stronger inhibition between neural representations of adjacent choices. Thus, the normalization can be realized within a local circuit, without making assumptions about the sensory input. 
Beck et al. (2008) proposed a model based on Bayesian formulation and predicted that the neuronal activity in LIP reflects the probability of the monkey's behavior. In accordance with that, we found that, in the eight-alternative task, the probability of choosing one incorrect target decays with increasing the angular distance between that and the correct target (Fig. 4C).

In a discrete network model for decision-making (Albantakis and Deco, 2009), the network remains in a symmetric steady state after motion onset until the firing rates for different neural pools diverge. It takes a longer time to break symmetry in the $2 \mathrm{~T}-90$ condition than in the $2 \mathrm{~T}-180$ condition because of stronger mutual excitation between competing neural pools. In contrast, here the difference in reaction time between the two conditions is attributable to strong similar-feature inhibition, which leads to a lower buildup rate of ramping activity in the 2T-90 condition. There are two more differences between the two models.

Because stronger excitation exists between the competing neural pools in the $2 \mathrm{~T}-90$ condition, they have a slightly higher firing rate before motion onset than in the $2 \mathrm{~T}-180$ condition (Fig. 3 in Albantakis and Deco, 2009). In our model, a stronger inhibition exists in the 2T-90 condition, and thus the neural pools have a slightly lower firing rate in the $2 \mathrm{~T}-90$ condition; this reduction persists throughout the dip. After the dip following motion onset, only the firing rate of the winning pool ramps up, whereas that of the other ramps down (Albantakis and Deco, 2009, their Fig. 3B). In our model, the dip is always followed by activity buildup for all neural pools located around each target's direction before their firing rates diverge (Fig. 3A). Our results are all consistent with the experimental observations (Churchland et al., 2008).

\section{Dopamine and structured inhibition}

We showed that the relative connectivity strength of oppositefeature versus similar-feature inhibition affects the tradeoff between speed and accuracy of decisions. Thus, the circuit can be optimized for distinct decision-making tasks by modulating synaptic strength. Our model has not addressed how the structure of inhibition dynamically changes over time to adapt to different decision-making tasks. It was found that similar-feature inhibition can be enhanced by dopamine administration (Rao et al., 2000; Gao and Goldman-Rakic, 2003; Williams and Castner, 2006). Possibly, a model that incorporates a dopaminergic reward system could be built in the future.

\section{References}

Abbott LF, Chance FS (2005) Drivers and modulators from push-pull and balanced synaptic input. Prog Brain Res 149:147-155. CrossRef Medline

Albantakis L, Deco G (2009) The encoding of alternatives in multiplechoice decision making. Proc Natl Acad Sci U S A 106:10308-10313. CrossRef Medline

Albright TD, Desimone R, Gross CG (1984) Columnar organization of directionally selective cells in visual area MT of the macaque. J Neurophysiol 51:16-31. Medline

Ardid S, Wang XJ, Compte A (2007) An integrated microcircuit model of attentional processing in the neocortex. J Neurosci 27:8486-8495. CrossRef Medline

Ardid S, Wang XJ, Gomez-Cabrero D, Compte A (2010) Reconciling coherent oscillation with modulation of irregular spiking activity in selective attention: gamma-range synchronization between sensory and executive cortical areas. J Neurosci 30:2856-2870. CrossRef Medline

Bartos M, Vida I, Frotscher M, Geiger JRP, Jonas P (2001) Rapid signaling at inhibitory synapses in a dentate gyrus interneuron network. J Neurosci 21:2687-2698. Medline

Beck JM, Ma WJ, Kiani R, Hanks T, Churchland AK, Roitman J, Shadlen MN,
Latham PE, Pouget A (2008) Probabilistic population codes for Bayesian decision making. Neuron 60:1142-1152. CrossRef Medline

Britten KH, Newsome WT (1998) Tuning bandwidths for near-threshold stimuli in area MT. J Neurophysiol 80:762-770. Medline

Brouwer GJ, Heeger DJ (2011) Cross-orientation suppression in human visual cortex. J Neurophysiol 106:2108-2119. CrossRef Medline

Churchland AK, Kiani R, Shadlen MN (2008) Decision-making with multiple alternatives. Nat Neurosci 11:693-702. CrossRef Medline

Compte A, Brunel N, Goldman-Rakic PS, Wang XJ (2000) Synaptic mechanisms and network dynamics underlying spatial working memory in a cortical network model. Cereb Cortex 10:910-923. CrossRef Medline

De Bruyn B, Orban GA (1988) Human velocity and direction discrimination measured with random dot patterns. Vision Res 12:1323-1335. Medline

Edin F, Klingberg T, Johansson P, McNab F, Tegnér J, Compte A (2009) Mechanism for top-down control of working memory capacity. Proc Natl Acad Sci U S A 106:6802-6807. CrossRef Medline

Funahashi S, Bruce CJ, Goldman-Rakic PS (1989) Mnemonic coding of visual space in the monkey's dorsolateral prefrontal cortex. J Neurophysiol 61:331-349. Medline

Furman M, Wang XJ (2008) Similarity effect and optimal control of multiplechoice decision making. Neuron 60:1153-1168. CrossRef Medline

Gao WJ, Goldman-Rakic PS (2003) Selective modulation of excitatory and inhibitory microcircuits by dopamine. Proc Natl Acad Sci U S A 100: 2836-2841. CrossRef Medline

Gilbert CD, Wiesel TN (1989) Columnar specificity of intrinsic horizontal and corticocortical connections in cat visual cortex. J Neurosci 9:24322442. Medline

Hol K, Treue S (2001) Different populations of neurons contribute to the detection and discrimination of visual motion. Vision Res 41:685-689. CrossRef Medline

Huk AC, Shadlen MN (2005) Neural activity in macaque parietal cortex reflects temporal integration of visual motion signals during perceptual decision making. J Neurosci 25:10420-10436. CrossRef Medline

Jazayeri M, Movshon JA (2007) A new perceptual illusion reveals mechanisms of sensory decoding. Nature 446:912-915. CrossRef Medline

Kaas JH (2012) Evolution of columns, modules, and domains in the neocortex of primates. Proc Natl Acad Sci U S A 109 [Suppl 1]:10655-10660. CrossRef Medline

Krimer LS, Goldman-Rakic PS (2001) Prefrontal microcircuits: Membrane properties and excitatory input of local, medium, and wide arbor interneurons. J Neurosci 21:3788-3796. Medline

Liu F, Wang XJ (2008) A common cortical circuit mechanism for perceptual categorical discrimination and veridical judgment. PLoS Comput Biol 4:e1000253. CrossRef Medline

Ma WJ, Beck JM, Latham PE, Pouget A (2006) Bayesian inference with probabilistic population codes. Nat Neurosci 9:1432-1438. CrossRef Medline

Markram H, Lübke J, Frotscher M, Roth A, Sakmann B (1997) Physiology and anatomy of synaptic connections between thick tufted pyramidal neurones in the developing rat neocortex. J Physiol 500:409-440. Medline

Martin KA (1988) The Wellcome Prize lecture. From single cells to simple circuits in the cerebral cortex. Q J Exp Physiol 73:637-702. Medline

Matsubara JA, Cynader MS, Swindale NV (1987) Anatomical properties and physiological correlates of the intrinsic connections in cat area 18. J Neurosci 7:1428-1446. Medline

Morrone MC, Burr DC, Maffei L (1982) Functional implications of crossorientation inhibition of cortical visual cells. I. Neurophysiological evidence. Proc R Soc Lond B Biol Sci 216:335-354. CrossRef Medline

Neri P, Levi D (2009) Surround motion silences signals from samedirection motion. J Neurophysiol 102:2594-2602. CrossRef Medline

Purushothaman G, Bradley DC (2005) Neural population code for fine perceptual decisions in area MT. Nat Neurosci 8:99-106. CrossRef Medline

Rao SG, Williams GV, Goldman-Rakic PS (1999) Isodirectional tuning of adjacent interneurons and pyramidal cells during working memory: Evidence for microcolumnar organization in PFC. J Neurophysiol 81:19031916. Medline

Rao SG, Williams GV, Goldman-Rakic PS (2000) Destruction and creation 
of spatial tuning by disinhibition: $\mathrm{GABA}_{\mathrm{A}}$ blockade of prefrontal cortical neurons engaged by working memory. J Neurosci 20:485-494. Medline

Ringach DL, Hawken MJ, Shapley R (1997) Dynamics of orientation tuning in macaque primary visual cortex. Nature 387:281-284. CrossRef Medline

Roe RM, Busemeyer JR, Townsend JT (2001) Multialternative decision field theory: A dynamic connectionist model of decision making. Psychol Rev 108:370-392. CrossRef Medline

Roerig B, Kao JPY (1999) Organization of intracortical circuits in relation to direction preference maps in ferret visual cortex. J Neurosci 19:RC44(1-5). Medline

Roitman JD, Shadlen MN (2002) Response of neurons in the lateral intraparietal area during a combined visual discrimination reaction time task. J Neurosci 22:9475-9489. Medline

Shadlen MN, Newsome WT (2001) Neural basis of a perceptual decision in the parietal cortex (area LIP) of the rhesus monkey. J Neurophysiol 86: 1916-1936. Medline

Tuckwell HC (1988) Introduction to theoretical neurobiology. Cambridge, UK: Cambridge UP.

Tversky A (1972) Elimination by aspects: a theory of choice. Psychol Rev 79:281-299. CrossRef
Wang XJ (2002) Probabilistic decision making by slow reverberation in cortical circuits. Neuron 36:955-968. CrossRef Medline

Weymouth FW (1958) Visual sensory units and the minimal angle of resolution. Am J Ophthalmol 46:102-113. Medline

Williams GV, Castner SA (2006) Under the curve: Critical issues for elucidating D1 receptor function in working memory. Neuroscience 139:263276. CrossRef Medline

Wong KF, Wang XJ (2006) A recurrent network mechanism of time integration in perceptual decisions. J Neurosci 26:1314-1328. CrossRef Medline

Wong KF, Huk AC, Shadlen MN, Wang XJ (2007) Neural circuit dynamics underlying accumulation of time-varying evidence during perceptual decision-making. Front Comput Neurosci 1:1-11. CrossRef Medline

Wörgötter F, Koch C (1991) A detailed model of the primary visual pathway in the cat: comparison of afferent excitatory and intracortical inhibitory connection schemes for orientation selectivity. J Neurosci 11:1959-1979. Medline

Yaniv I, Schul Y (1997) Elimination and inclusion procedures in judgment. J Behav Decis Making 10:211-220. CrossRef

You H and Wang DH (2013) Dynamics of multiple-choice decision making. Neural Comput 25:2108-2145. CrossRef Medline 